\title{
Intra-carotid arterial transfusion of circulatory- derived autologous endothelial progenitor cells in rodent after ischemic stroke - evaluating the impact of therapeutic time points on prognostic outcomes
}

Kun-Chen Lin

Chang Gung Memorial Hospital Kaohsiung Branch

Han-Tan Chai

Chang Gung Memorial Hospital Kaohsiung Branch

Kuan-Hung Chen

Chang Gung Memorial Hospital Kaohsiung Branch

\section{Pei-Hsun Sung}

Chang Gung Memorial Hospital Kaohsiung Branch

John Y. Chiang

National Sun Yat-sen University

Pei-Lin Shao

Asia University

Chi-Ruei Huang

Chang Gung Memorial Hospital Kaohsiung Branch

Yi-Chen Li

Chang Gung Memorial Hospital Kaohsiung Branch

\section{Sheung-Fat Ko}

Chang Gung Memorial Hospital Kaohsiung Branch

Hon-Kan Yip ( $\square$ han.gung@msa.hinet.net)

Chang Gung Memorial Hospital Kaohsiung Branch https://orcid.org/0000-0002-6305-5717

\section{Research}

Keywords: ischemic stroke, endothelial progenitor cells, angiogenesis, neurological function

Posted Date: March 19th, 2020

DOI: https://doi.org/10.21203/rs.2.23279/v2 
License: (c) (i) This work is licensed under a Creative Commons Attribution 4.0 International License. Read Full License

Version of Record: A version of this preprint was published at Stem Cell Research \& Therapy on June 5th, 2020. See the published version at https://doi.org/10.1186/s13287-020-01739-y. 


\section{Abstract}

Background: This study tested the optimal time point for left intra-carotid arterial (LICA) administration of circulatory-derived autologous endothelial progenitor cells (EPCs) for improving the outcome in rat after acute ischemic stroke (IS).

Methods and Results: Adult-male SD rats $(\mathrm{n}=70)$ were equally categorized into group 1 (sham-operated control), group 2 (IS), group 3 (IS+EPCs/1.2×106 cells/by LICA administration 3h after IS), group 4 (IS+EPCs/LICA administration post-day-3 IS), group 5 (IS+EPCs/LICA administration post-day-7 IS), group 6 (IS+EPCs/LICA administration post-day-14 IS) and group 7 (IS+EPCs/LICA administration post-day-28 IS). The brain-infarct volume (BIV) (at day 60/MRI) was lowest in group 1, highest in group 2 and significantly progressively increased from groups 3 to 7, whereas among the IS animals, the neurological function was significantly preserved in groups 3 to 6 than in groups 2 and 7 post-day- 60 IS (all $p<0.0001$ ). By day 60 , the endothelial cell markers at protein and cellular levels, and number of small vessels exhibited an opposite pattern of BIV among the groups (all $p<0.0001$ ). The protein and cellular levels of inflammation, and protein levels of oxidative stress, autophagy and apoptosis, were highest in group 2, lowest in group 1 and progressively increased from groups 3 to 7 (all $p<0.0001$ ). The angiogenesis biomarkers at protein and cellular levels were significantly progressively increased from groups 1 to 3 , then significantly progressively decreased from groups 4 to 7 (all $p<0.0001)$.

Conclusion: Early EPC administration provided better benefits on improving functional/image/molecularcellular outcomes after acute IS in rat.

\section{Introduction}

Although divergent etiologies cause stroke, atherosclerotic intracranial arterial stenosis/occlusion remains one of the common causes of ischemic stroke (IS) worldwide [1-6] and is associated with a high risk of frequently recurrent IS once IS developed [7-9]. Surprisingly, while the epidemiology, etiologies, mechanisms, classification, and prognostic outcomes of IS have been widely investigated for several decades, a safe and effective treatment strategy for the majority of patients after acute IS has not been fully developed [10-14]. Hence, finding a safe and effective therapeutic option for IS patients is an important issue.

Our previous studies have shown that circulating level of EPCs was notably frequently increased in patients after acute IS $[15,16]$, suggesting acute IS event would stimulate the EPCs mobilization from bone marrow to circulation. Additionally, our studies $[15,16]$ have further identified that an increase in circulating level of EPCs was strongly associated with favorable clinical outcomes after IS $[15,16]$. Importantly, these findings demonstrated that circulating level of EPCs can serve as a useful biomarker for stratification of IS patients into high- and low-risk groups with respect to future outcomes $[15,16]$. Based on the findings from our studies $[15,16]$, we subsequently performed an acute IS model in rodent and treated by intra-carotid artery administration of circulatory derived autologous EPCs [17]. Intriguingly, 
the results revealed that this therapy significantly reduced the brain infarct size and improved neurological dysfunction after acute IS in rodent mainly through enhancement of angiogenesis and neurogenesis, and reduction of inflammation, oxidative stress and cellular apoptosis [17]. The results of these studies [15-17] encouraged us to perform a phase I clinical trial of intra-carotid artery transfusion of circulatory derived autologous EPCs into brain infarct area in old IS stroke patients [18]. The results of our study displayed that EPC therapy was safe. However, the neurological and neuro-psychiatric functions as well as the brain perfusion status were found to have only minor improvement in those patients [18]. The results of these aforementioned unmet needs from our study [18] raise the consideration of a large randomized placebo-controlled trial to test the safety and efficacy of EPC therapy for patients after acute IS. In light of the above observation, an animal model of IS study, before applying for a clinical trial, focusing on the optimal time point for administration of EPCs that would offer the greatest benefit and the minimal side effect is required. Accordingly, this study using a rat model of acute IS tried to find out the best time point of circulatory derived autologous EPC administration for reducing the brain infarct volume (BIV)/brain infarct area (BIA) and improving the neurological outcome in IS rat.

\section{Materials And Methods}

\section{Procedure and protocol of acute IS induction}

The protocol and procedure for the experimental model of acute IS have been described in our previous studies $[17,19]$. In detail, each animal was anesthetized by $2 \%$ inhalational isoflurane in a supine position on a warming pad $\left(37^{\circ} \mathrm{C}\right)$. After exposure of the left common carotid artery (LCCA) through a transverse neck incision, a small arteriotomy was performed on the LCCA through which silicon rubber-coated monofilament (filament size 4-0, diameter $0.185 \mathrm{~mm}$, length $30 \mathrm{~mm}$; Co Ltd. AMBO) was carefully advanced into the distal left internal carotid artery for occlusion of the left middle cerebral artery, causing brain ischemia and infarction of the corresponding territory area. The nylon monofilament was removed 50 mins after occlusion, followed by closure of the muscle and skin in layers. The animals were then cared for in a portable animal intensive care unit (ThermoCare ${ }^{\circledR}$ ) with food and water for 24 hours.

\section{Corner test for assessment of neurological function prior to and after IS induction}

The sensorimotor functional test (corner test) was conducted for each rat at baseline and on days $0,3,7$, 14,28 and 60 after acute IS induction as we previously described [17, 19]. In detail, the rat could walk through a tunnel and then turn into a 60-degree corner. To exit the corner, the rat could turn either left or right. The results were recorded by a technician blinded to the study design. This test was repeated 10 to 15 times with at least 30 seconds between each trial. We recorded the number of right and left turns from 10 successful trials for each animal and used the results for statistical analysis. All 10 animals in each group went through this neurological functional test.

\section{Animal grouping and treatment protocol}


Animals were housed in an Association for Assessment and Accreditation of Laboratory Animal Care International-approved animal facility in our hospital, with controlled temperature and light cycles $\left(24^{\circ} \mathrm{C}\right.$ and 12/12 light/dark cycle). Pathogen-free, adult male Sprague-Dawley (SD) rats $(n=70)$ weighing around 300-325g (Charles River Technology, BioLASCO Taiwan Co. Ltd., Taiwan) were utilized in the present study. Among the animals, the sham-operated control (SC) was first randomized. After acute IS induction procedure, the animals were further randomized into acute ischemic stroke (IS), acute IS + EPCs (1.2 x $10^{6}$ cells by left ICA administration at $3 \mathrm{~h}$ after IS) $\left(\mathrm{EPC}^{3 \mathrm{~h}}\right)$, acute IS + EPCs $\left(1.2 \times 10^{6}\right.$ cells by left ICA administration at day 3 after IS) $\left(E^{2} C^{3 d}\right)$, acute IS + EPCs $\left(1.2 \times 10^{6}\right.$ cells by left ICA administration at day 7 after IS) $\left(\right.$ EPC $\left.^{7 d}\right)$, acute IS + EPCs $\left(1.2 \times 10^{6}\right.$ cells by left ICA administration at day 14 after IS) $\left(E^{2} C^{14 d}\right)$ and acute IS + EPCs $\left(1.2 \times 10^{6}\right.$ cells by left ICA administration at day 28 after IS (EPC $\left.{ }^{28 d}\right)$, respectively. At $1.2 \mathrm{~h}$ after removing the silicon rubber-coated monofilament, the EPCs were slowly transfused into the LCCA.

The number of operated-related (i.e., included IS induction and EPC administration) dead animals in SC, IS, EPC ${ }^{3 h}, E^{3 d}, E^{3 d}, C^{7 d}, E^{14 d}, E^{128 d}$ were, 0, 4, 3, 5, 4, 3, and 5, respectively. On the other hand, no any animal was excluded due to unsuccessful induction.

The dosage of EPCs administered in the present study was based on our previous report [17]. For the molecular-cellular examination, six animals in each group were utilized.

Ibuprofen $(15 \mathrm{mg} / \mathrm{kg} /$ day) dissolved in drinking water was orally administered for the pain management for the animals for 3 to 7 days after the IS-induced procedure.

Animals in each group were euthanized by day 60 after IS induction. Prior to euthanization, the animals were anesthetized by $5 \%$ of Isoflurane mixed with $100 \%$ of oxygen $3 \mathrm{~L} / \mathrm{min}$, followed by drawing the blood $10 \mathrm{~mL}$ from abdominal aorta to induce hypovolemic shock and death. Finally, the wound was sutured after the brain specimen was harvested from each animal.

\section{Peripheral blood was collected and cultured for EPCs}

The procedure and protocol for EPC isolation and culture were based on our previous reports [17] with some modifications. In brief, animals in groups $\mathrm{EPC}^{3 \mathrm{~h}}, \mathrm{EPC}^{3 \mathrm{~d}}, \mathrm{EPC}^{7 \mathrm{~d}}$ and $\mathrm{EPC}^{14 \mathrm{~d}}$ were anesthetized with inhalational $2.0 \%$ isoflurane, i.e., by day 21 at $\mathrm{EPC}^{3 \mathrm{~h}}$, by day 18 at $\mathrm{EPC}^{3 \mathrm{~d}}$, by day 14 at $\mathrm{EPC}^{\mathrm{d} 7}$ and by day 7 at EPC ${ }^{14 d}$ prior to IS induction and by day 7 after IS induction at EPC ${ }^{28 d}$ to collect peripheral blood for culturing EPCs (refer to the supplementary Figure 1). Isolated mononuclear cells from peripheral blood were cultured in a 100-mm diameter dish with $10 \mathrm{~mL}$ DMEM culture medium containing 10\% FBS for 21 days. Based on our previous method [17], flow cytometric analysis was performed for identification of cellular characteristics (i.e., EPC surface markers) after cell-labeling with appropriate antibodies on day 21 of cell cultivation prior to cell therapy (i.e., EPCs were transfused into LCCA on day 21 after cell culture) (refer to the supplementary Figure 2). 
Rats in groups 1 and 2 were anesthetized with inhalational 2.0\% isoflurane and only received an identical procedure of peripheral blood sampling without further culture.

\section{Procedure and protocol of brain magnetic resonance imaging (MRI) study}

The procedure and protocol have been described in our previous report [17]. Magnetic resonance imaging (MRI) was performed at day 60 and after acute IS induction. Briefly, during MRI measurements, mice were anesthetized by $2 \%$ inhalational isoflurane with room air and placed in an MRI-compatible holder (Biospec 94/20, Bruker, Ettingen, Germany). Rectal temperature and respiration were monitored throughout the procedure to ensure normal physiological conditions were maintained. MRI data were collected using a Varian 9.4T animal scanner (Biospec 94/20, Bruker, Ettingen, Germany) with a rat surface array. The MRI protocol consisted of 40 T2-weighted images. Forty continuous slice locations were imaged with a field-of-view of $30 \mathrm{~mm} \times 30 \mathrm{~mm}$ and an acquisition matrix dimension of $256 \times 256$ and slice thickness of $0.5 \mathrm{~mm}$. The repetition time (TR) and echo time (TE) for each fast spin-echo volume were $4200 \mathrm{~ms}$ and $30 \mathrm{~ms}$, respectively. A custom software, ImageJ (1.43i, NIH, USA), was used to process the region of interest (ROI). Planimetric measurements of images from MRI T2 were performed to calculate the stroke volumes of cortex. For brain MRI examination, four randomized animals in each group were utilized. The animals were euthanized on day 60 after brain MRI examination and the brain tissues were harvested for individual study.

\section{Immunofluorescent (IF) staining of brain specimens}

The procedure and protocol for IF staining have been reported in our previous studies [17-19]. For IF staining, rehydrated paraffin sections were first treated with $3 \% \mathrm{H}_{2} \mathrm{O}_{2}$ for 30 minutes and incubated with Immuno-Block reagent (BioSB, Santa Barbara, CA, USA) for 30 minutes at room temperature. Sections were then incubated with primary antibodies specifically against CD31 (1:100, Bio-Rad), von Willebrand factor (vWF) (1:200, Merck Millipore), NeuN (1:1000, Millipore, Billerica, MA, USA), stromal cell-derived factor [(SDF)-1a (1:100, Santa Cruz Biotechnology), vascular endothelial growth factor (VEGF) (1:400, Abcam), CXCR4 (1:200, Thermo Fisher Scientific), microglial cell (1:100, Abcam, Cambridge, UK), and Glial fibrillary acidic protein (GFAP) $\left(1: 500\right.$, Dako) at $4^{\circ} \mathrm{C}$ overnight. Alexa Fluor488, Alexa Fluor568, or Alexa Fluor594-conjugated goat anti-mouse or rabbit IgG were used to localize signals. Sections were finally counterstained with DAPI and observed with a fluorescent microscope equipped with epifluorescence (Olympus IX-40).

Three brain sections were analyzed for each rat. For quantification, three randomly selected high-power fields (HPFs; $\mathrm{x} 400$ for IF study) were analyzed in each section. The mean number of positively-stained cells per HPF for each animal was then determined by summation of all numbers divided by 9 .

\section{Western blot analysis of brain specimens}

Equal amounts $(50 \mathrm{mg}$ ) of protein extracts were loaded and separated by SDS-PAGE using $12 \%$ acrylamide gradients. After electrophoresis, the separated proteins were transferred electrophoretically to 
a polyvinylidene difluoride (PVDF) membrane (Amersham Biosciences, Amersham, UK). Nonspecific sites were blocked by incubation of the membrane in blocking buffer [5\% nonfat dry milk in T-TBS (TBS containing $0.05 \%$ Tween 20$)$ ] overnight. The membranes were incubated with monoclonal antibodies against CXCR4 (1:1000, Abcam), SDF-1a (1:1000, Cell Signalimg, Danvers, MA, USA), VEGF (1:1000, Abcam), CD31 (1:1000, Abcam), von Willebrand factor (vWF) (1:1000, Abcam), NOX-1 (1:1500, SigmaAldrich), NOX-2 (1:500, Sigma-Aldrich), matrix metalloproteinase (MMP)-9 (1:3000, Abcam), tumor necrosis factor alpha (TNF-a; 1:1000, Cell Signaling), nuclear factor (NF)-kB (1:1000, Abcam), interleukin (IL)-1ß (1:1000, Cell Signaling), caspase 3 (1:3000, Abcam), mitochondrial Bax (1:1000, Abcam), beclin-1 (1:1000, Cell Signaling), Atg5 (1:1000, Cell Signaling), LCB3-II (1:2000, Abcam) and LCB3-I (1:2000, Abcam) for $1 \mathrm{~h}$ at room temperature. Horseradish peroxidase-conjugated anti-rabbit or anti-mouse immunoglobulin IgG (1:2000, Cell Signaling) was used as a second antibody for $1 \mathrm{~h}$ at room temperature. The washing procedure was repeated eight times within $1 \mathrm{~h}$, and immunoreactive bands were visualized by enhanced chemiluminescence (ECL; Amersham Biosciences) and exposed to Medical X-ray film (FUJI). For quantitation, ECL signals were digitized using Labwork software (UVP, Waltham, MA, USA). A standard control sample was loaded on each gel.

\section{Vessel density in peri-infarct area}

IHC staining of small blood vessels was performed with a-SMA (1:400) as primary antibody at room temperature for $1 \mathrm{~h}$, followed by washing with PBS three times. Ten minutes after the addition of antimouse-HRP conjugated secondary antibody, the tissue sections were washed with PBS three times. Then 3,3' diaminobenzidine (DAB) $(0.7 \mathrm{gm} /$ tablet) (Sigma-Aldrich) was added, followed by washing with PBS three times after one minute. Finally, hematoxylin was added as a counter-stain for nuclei, followed by washing twice with PBS after one minute. Three brain sections were analyzed in each rat. For quantification, three randomly selected HPFs (200x) were analyzed in each section. The mean number per HPF for each animal was then determined by summation of all numbers divided by 9 .

\section{Statistical analyses}

Quantitative continuous data are expressed as mean \pm SD. Statistical analysis was performed for continuous variables among groups by one-way ANOVA followed by Bonferroni multiple-comparison post hoc test for the between-group comparison. All analyses were conducted using SAS statistical software for Windows version 8.2 (SAS institute, Cary, NC, USA). A probability value $<0.05$ was considered statistically significant.

\section{Results}

\section{The brain infarct volume (BIV) at day 60 after IS and the time courses of corner test after acute IS (Figures 1 and 2)}

At day 60 after acute IS, the brain MRI demonstrated that the BIV was lowest in SC, highest in IS and significantly and progressively increased from EPC ${ }^{3 \mathrm{~h}}, \mathrm{EPC}^{3 \mathrm{~d}}, \mathrm{EPC}^{7 \mathrm{~d}}, \mathrm{EPC}^{14 \mathrm{~d}}$ to $\mathrm{EPC}^{28 \mathrm{~d}}$ (Fig. 1). 
The neurological function did not differ at baseline among seven groups. However, at day 3 after acute IS, the neurological function was significantly impaired in all the IS groups regardless with or without treatment than in SC, but it showed no difference among the six IS groups (Fig. 2).

On the other hand, at day 7 after IS, this parameter was significantly improved in EPC ${ }^{3 \mathrm{~h}}$ and $E P C^{3 \mathrm{~d}}$ than in IS, EPC $7 \mathrm{~d}$ to $E P C^{28 d}$, but it did not differ between groups $\mathrm{EPC}^{3 \mathrm{~h}}$ and $\mathrm{EPC}^{3 \mathrm{~d}}$ or among IS, EPC ${ }^{7 d}, \mathrm{EPC}^{14 \mathrm{~d}}$ and $\mathrm{EPC}^{28 \mathrm{~d}}$ (Fig. 2).

Additionally, at days 14 after IS, the neurological defect was lowest in SC, highest in IS, EPC ${ }^{14 d}$ and $E P C^{28 d}$ and significantly higher in $\mathrm{EPC}^{7 \mathrm{~d}}$ than in $\mathrm{EPC}^{3 \mathrm{~h}}$ and $E P C^{3 \mathrm{~d}}$, but it showed no difference between $\mathrm{EPC}^{3 \mathrm{~h}}$ and $\mathrm{EPC}^{3 \mathrm{~d}}$ or among IS, EPC ${ }^{14 \mathrm{~d}}$ and EPC ${ }^{28 \mathrm{~d}}$ (Fig. 2)

Furthermore, by day 28 after IS, the neurological defect was significantly lower in SC, $\mathrm{EPC}^{3 \mathrm{~h}}$ and $\mathrm{EPC}^{3 \mathrm{~d}}$ than in other groups and significantly lower in EPC ${ }^{7 d}$ and EPC ${ }^{14 d}$ than in IS and EPC ${ }^{28 d}$, but it showed no difference among SC, $\mathrm{EPC}^{3 \mathrm{~h}}$ and $\mathrm{EPC}^{3 \mathrm{~d}}$, between $\mathrm{EPC}^{7 \mathrm{~d}}$ and $\mathrm{EPC}^{14 \mathrm{~d}}$ or between IS and $\mathrm{EPC}^{28 \mathrm{~d}}$ (Fig. 2).

Finally, at day 60 after IS, the neurological defect was lowest in SC, EPC ${ }^{3 \mathrm{~h}}$ and $\mathrm{EPC}^{3 \mathrm{~d}}$, highest in IS and $\mathrm{EPC}^{28 \mathrm{~d}}$, and significantly lower in $\mathrm{EPC}^{\mathrm{d} 7}$ than in $\mathrm{EPC}^{\mathrm{d} 14}$, but it did not differ among the SC, $\mathrm{EPC}^{3 \mathrm{~h}}$ and $\mathrm{EPC}^{3 \mathrm{~d}}$ or between IS and $\mathrm{EPC}^{28 \mathrm{~d}}$ (Fig. 2).

\section{The expressions of angiogenesis biomarkers in protein and cellular levels in BIA at day 60 after acute IS (Figures 3, 4, 5 and 6)}

First, we performed the Western blotting for identifying the protein level of angiogenesis biomarkers. The results demonstrated that the protein expressions of CD31 and VWF, two indicators of endothelial functional integrity, were highest in SC, lowest in IS, significantly higher in $E P C^{3 \mathrm{~h}}$ and $E P C^{3 \mathrm{~d}}$ than in $E P C^{7 d}, E P C^{14 d}$ and $E P C^{28 d}$, significantly higher in $E P C^{3 h}$ than in $E P C^{3 d}$, but they did not differ among the $E^{E P C}{ }^{7 d}, E^{2} C^{14 d}$ and EPC ${ }^{28 d}$ (Fig. 3). Additionally, the protein expressions of VEGF, CXCR4 and SDF-1a, three indices of angiogenesis factors, were lowest in $\mathrm{SC}$ and highest in $\mathrm{EPC}^{3 \mathrm{~h}}$, significantly higher in

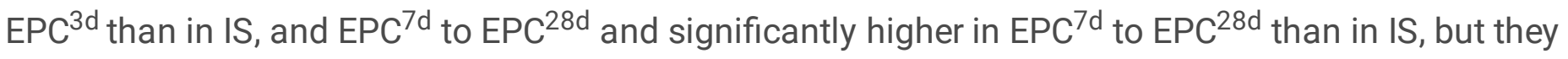
showed no difference among the groups $\mathrm{EPC}^{7 \mathrm{~d}}$ to $\mathrm{EPC}^{28 \mathrm{~d}}$ (Fig. 3).

Next, we performed IF microscope for assessment of endothelial cell surface markers. The results displayed that the cellular expressions of CD31 and vWF were highest in SC, lowest in IS, and significantly progressively reduced from $\mathrm{EPC}^{3 \mathrm{~h}}$ to $E P C^{28 \mathrm{~d}}$, but they showed no difference between the $E C^{14 d}$ and $\operatorname{EPC}^{28 d}$ (Fig. 4).

Additionally, the cellular expressions of SDF-1a and CXCR4 were highest in EPC ${ }^{3 \mathrm{~h}}$, lowest in SC, significantly higher in $\mathrm{EPC}^{3 \mathrm{~d}}$ than in IS, EPC ${ }^{7 \mathrm{~d}}$ to $E P C^{28 \mathrm{~d}}$, significantly higher in $\mathrm{EPC}^{7 \mathrm{~d}}$ to $I S, E P C^{14 \mathrm{~d}}$ and $E P C^{28 d}$ and significantly higher in EPC ${ }^{14 d}$ and EPC ${ }^{28 d}$ than in IS, but they were similar between EPC ${ }^{14 d}$ 
and $\mathrm{EPC}^{28 \mathrm{~d}}$, suggesting an intrinsic response to ischemic stimulation that was significantly upregulated by EPCs treatment (Fig. 5).

To elucidate the angiogenesis/neovascularization in the peri-infarct region, we performed IHC staining (i.e., a-SMA stain). As expected, the number of small vessel (i.e., diameter $\leq 25 \mu \mathrm{M}$ ) exhibited a similar pattern to that of the protein levels of CD31 and vWF among the groups (Fig. 6).

The protein expressions of inflammatory, oxidative stress, autophagic and apoptotic biomarkers in BIA at day 60 after acute IS (Figures 7 and 8 )

The protein expressions of IL-1ß, TNF- - , NF-KB and MMP-9, four indicators of inflammation, were lowest in $S C$ and highest in IS, significant lower in EPC ${ }^{3 d}$ and more significantly lower in $E P C^{3 \mathrm{~h}}$ than in $E P C^{7 d}$ to $\mathrm{EPC}^{28 \mathrm{~d}}$, but they exhibited no difference among the EPC ${ }^{7 \mathrm{~d}}$ to EPC ${ }^{28 \mathrm{~d}}$ (Fig. 7).

Additionally, the protein expressions of NOX-1, NOX-2, oxidized protein and p22phox, four indicators of oxidative stress, displayed an identical pattern of inflammation among the groups (Fig. 7). Furthermore, the protein expressions of Beclin-1, Atg-5 and ratio of LC3B-II/LC3B-I, three indicators of autophagic biomarkers, exhibited an identical pattern of inflammation among the groups (Fig. 8).

Moreover, the protein expressions of mitochondrial-Bax and cleaved PARP, two indices of apoptosis, showed an identical pattern to that of inflammation among the groups (Fig. 8).

Interestingly, previous study showed that cleaved caspase 3 expression was predominantly associated with cellular responses to stroke such as reactive astrogliosis and the infiltration of macrophages [20]. In the present study, we also found that the protein expression of cleaved caspase 3 was identical to the inflammation among the groups (Fig. 8), implicating that this parameter might not be necessarily a biomarker of apoptosis after IS.

\section{Inflammatory cell expressions of GFPA+ and microglial cells in BIA at day 60 after acute IS (Figure 9)}

The results of IF microscopic findings demonstrated that the expressions of GFAP and microglial cells, two indicators of inflammatory cells specifically situated in brain tissue, was lowest in SC, highest in IS and significantly and progressively increased from $\mathrm{EPC}^{3 \mathrm{~h}}$ to $\mathrm{EPC}^{28 \mathrm{~d}}$, but it displayed no difference between $\mathrm{EPC}^{7 \mathrm{~d}}$ and $\mathrm{EPC}^{14 \mathrm{~d}}$.

\section{Discussion}

This study which investigated the appropriate time point of intra-carotid administration of circulatory derived autologous EPCs for improving the neurological function and reducing the BIV yielded several preclinical striking implications. First, despite the difference in the degree of preservation of neurological function and brain architecture, the EPCs therapy at time intervals $\leq 14$ days after IS still significantly preserved the neurological function and integrity of brain architecture in rat acute IS. Second, through 
different time intervals of administrating EPCs for rat after acute IS, this study identified that the optimal time point of EPC therapy for improving the neurological function and reducing the infarct zone was as early as possible (i.e., at $3 \mathrm{~h}$ after acute IS).

Intra-carotid administration of circulatory EPCs significantly improved neurological outcome and reduced the brain infarct size in rat after acute IS having been well recognized by our previous study [17]. Surprisingly, there is currently seldom data available to address the optimal timing of administration of EPCs after acute IS. In the current study, time courses of EPCs therapy for identifying the most appropriate time point was done in rat IS model. The most important finding in the present study was that 3 hours after acute IS was the best timing for EPC administration to improve the neurological function and preserve brain architecture. Additionally, our result also observed therapeutic window at $\leq 3$ days upon cell administration also effectively preserved BIV and neurological function. Of importance was that all the parameters in molecular-cellular levels were consistent with the findings of brain MRI and corner test (i.e., neurological functional measurement). In this way, our findings clearly explained that EPC treatment was not only successful for the brain repair but also successful for the neuroprotection in rodent after acute IS. Accordingly, our finding, in addition to supporting the finding of our previous study [5], highlights the common concept that cell therapy should be performed "as early as possible" for ischemia-related organ dysfunction.

Interestingly, one previous study from Boltze $\mathrm{J}$, et al. has demonstrated that administration of human umbilical cord blood mononuclear cells (hUCB MNCs) at 4, 24, or $72 \mathrm{~h}$ effectuated a significant improvement of functional defects in rodent [21]. Therefore, their results for the first time indicate a time window of therapeutic hUCB MNC application of at least $72 \mathrm{~h}$. In this way, our findings were consistent with the findings from Boltze $\mathrm{J}$, et al [21].

Recently, we have performed a phase I clinical trial by utilizing the circulatory derived autologous CD34+ cells to treat those old IS patients [18]. Interestingly, although the safety of the cell therapy was confirmed [18], the therapeutic impact on improving the neurological and recognizing outcomes regrettably left much to be desired, attributing mainly to late treatment. Some previous clinical trials have also demonstrated that cell therapy for patients after acute myocardial infarction (AMI) failed to improve the heart function and clinical outcome $[22,23]$. In further, when we carefully analyzed the results from these clinical trials [22, 23], a commonality of late administration of stem cells to patients after AMI was uncovered. An essential finding in the present study was that as we serially tested the time points of administration of EPCs at $3 \mathrm{~h}$ and days 3, 7, 14 and 28 in rat after acute IS, the later the EPC administration, the lesser effective degree of neurological improvement was found in the present study. Accordingly, our finding, in addition to explaining why those clinical studies $[18,22,23]$ failed to prove the effectiveness of stem cell therapy for the improvement of the left ventricular (LV) function in patients after AMI, supports the common concept that only early administration of stem cells is the fundamentally mechanistic basis for improving ischemia-related organs, especially in setting of acute IS and AMI. 
The underlying mechanisms for improving the ischemia-related LV dysfunction after the cell therapy have been well recognized mainly due to the angiogenesis and neovascularization, chemokine/paracrine effects, stem cell homing, anti-inflammatory response and immunomodulation but they are less likely due to the implanted stem cells to differentiate into the cardiomyocytes [24-26]. Of these factors, the angiogenesis is one of the most important factors for improving the ischemia-related organ dysfunction $[16,17,24-26]$. An essential finding in the present study was that the molecular-cellular levels of angiogenesis parameters were markedly upregulated after IS stroke (i.e., due to an intrinsic response) and further markedly upregulated by EPC treatment, especially in the time point of early administration of EPCs to the animals after acute IS. Of particular importance was that increases of angiogenesis biomarker were significantly associated with improving neurological function and reducing the brain infarct zone in those animals receiving early EPC treatment. Our findings were consistent with those of previous studies $[16,17,24-26]$.

A principal finding in the present study was that the inflammatory, oxidative-stress, apoptotic and autophagic biomarkers were substantially increased in acute IS animals than in those of SC animals. Additionally, the neurological impairment and brain infarct region were remarkably increased in these acute IS animals. Intriguingly, links between upregulations of inflammation, oxidative stress, apoptosis and autophagic biomarkers and impaired ischemia-related organ dysfunction have been fully investigated by the previous experimental studies [17, 19, 24-28]. Along this line of thinking, our findings in addition to corroborating with the findings of previous studies [17, 19,24-28], could, at least in part, explain why the neurological status and BIV were notably deteriorated in those of acute IS animals without treatment. However, these molecular-cellular perturbations were significantly reversed in IS animals after receiving the early EPC treatment, once again suggesting that EPC treatment in an appropriate time point could effectively improve outcome in setting of acute IS. Accordingly, our preclinical findings may provide clinically relevant information and encourage us to utilize EPC therapy for those acute IS patients, especially when they have poor response or are refractory to conventional therapy.

In the present study, we identified that EPC therapy for the rodent at $3 \mathrm{~h}$ after acute IS was the ideal time for preservation of BIV and neurological function. However, it is impractical to apply the autologous EPCs to the patients within $3 \mathrm{~h}$ after IS not only due to the time is not enough for the well preparation of EPCs but also due to there's a relatively high potential risk for the patients at this time point to receive the treatment. On the other hand, previous has demonstrated that cryopreservation of cells would reduce the efficacy [29]. Additionally, ageing and senescence effects due to cryopreservation [30] that could also contribute to negative or neutral results [31]. Accordingly, when hemopoietic-derived stem cell therapy is taken into consideration for acute IS patients, minimal manipulation (i.e., cells without cultivation) method to collect the circulatory-derived/bone marrow-derived autologous CD34+ cells would be more practical and suitable for the IS patients in our daily clinical practice.

\section{Study limitation}


Investigators have previously suggested that functional recovery after an experimental stroke should be assessed by multiple behavioral tests to prevent false-positive results [32]. In the present study, only a sensorimotor function (i.e., corner test) applied might not be optimal for long-term assessment of functional deficits in stroke, suggesting that selecting an appropriate task or a battery of tasks should be necessary in our study. Second, microembolisms and decreased cerebral blood flow during intra-arterial delivery of allogeneic bone marrow mesenchymal stem cells has been reported by previously experimental study [33]. However, this study did not evaluate the possibility of microembolisms after EPCs transplantation that could cause the complication of embolic events and concomitant new infarct zone. Third, there was a reduction of BIV in groups receiving cell therapy at days 14 and 28 by brain MRI finding. Generally, the infarct is fully developed around day 14 , so any BIV reduction at EPC ${ }^{28 d}$ group compared to that of the controls might be explained by (1) lesion size differences at baseline (2) less likely, any type of tissue regrowth, (3) or due to the angiogenesis and tissue regeneration in ischemic boundary zone (i.e., cell at risks) after EPC therapy (refer to Figure 10, our proposed mechanism based on our findings). Finally, despite extensive works were done in the present study, the exactly underlying mechanisms of improving the outcomes after EPC therapy for acute IS remains uncertain. Based on the results of the present study, Figure 10 provides the schematically proposed mechanism for the impact of EPC therapy on mammalians after acute IS.

In conclusion, the results of the present study proved that early administration of EPCs for acute IS rodent would provide the best effect on improving the neurological outcome and protecting the brain architecture against acute IS damage.

\section{Abbreviations}

AMI: Acute myocardial infarction; Brain Infarct Area (BIA); BIV: Brain infarct volume; DMEM: Dulbecco's modified Eagle's medium; ECL: Enhanced chemiluminescence; EPC: Endothelial progenitor cells; FBS: Fetal bovine serum; GFAP: Glial fibrillary acidic protein; HPFs: High-power fields; ICA: Intra-carotid arterial; IF: Immunofluorescent; IHC: Immunohistochemistry; IS: Ischemic stroke; LCCA: Left common carotid artery; LV: Left ventricular; MMP: Matrix metalloproteinase; MRI: Magnetic resonance imaging; PARP: Poly (ADP-ribose) polymerase, PVDF: Polyvinylidene difluoride; SDF-1a: Stromal cell-derived factor; SDS-PAGE: Sodium dodecyl sulfate-Polyacrylamide gel electrophoresis; TBS: Tris-buffered saline; TE: Echo time; TR: Repetition time; VEGF: Vascular endothelial growth factor; vWF: von Willebrand Factor;

\section{Declarations}

\section{Acknowledgements}

This work was supported by a program grant from National Science Council, Taiwan (MOST 107-2314-B182A-056 -MY3). We thank Dr. John Y. Chiang for critical review for the manuscript. 


\section{Author Contributions}

Investigation, Kun-Chen Lin, Han-Tan Chai, Kuan-Hung Chen, Pei-Hsun Sung, Pei-Lin Shao, Chi-Ruei Huang, Yi-Chen Li, Sheung-Fat Ko; Methodology, Pei-Lin Shao and Sheung-Fat Ko; Supervision, SheungFat Ko and Hon-Kan Yip; Writing - review \& editing, John Y. Chiang and Hon-Kan Yip.

\section{Funding}

This study was supported by a program grant from National Science Council, Taiwan (MOST 107-2314-B182A-056-MY3).

\section{Availability of data and materials}

The data that support the findings of this study are available from the corresponding authors upon reasonable request.

\section{Ethics approval and consent to participate}

All animal experimental procedures were approved by the Institutional Animal Care and Use Committee at Kaohsiung Chang Gung Memorial Hospital (Affidavit of Approval of Animal Use Protocol No.

2017110201) and performed in accordance with the Guide for the Care and Use of Laboratory Animals, $8^{\text {th }}$ edition (NIH publication No. 85-23, National Academy Press, Washington, DC, USA, revised 2011).

\section{Consent for publication}

Not applicable.

\section{Conflicts of interest}

All authors have read the journal's policy on disclosure of potential conflicts of interest and the journal's authorship agreement. The authors declare that they have no conflicts of interest. The article has been reviewed by and approved by all named authors. 


\section{References}

[1] Barnett HJ, Gunton RW, Eliasziw M, et al. Causes and severity of ischemic stroke in patients with internal carotid artery stenosis. JAMA 2000; 283: 1429-1436.

[2] Barnett HJ, Taylor DW, Eliasziw M, et al. Benefit of carotid endarterectomy in patients with symptomatic moderate or severe stenosis. North American Symptomatic Carotid Endarterectomy Trial Collaborators. N Engl J Med 1998; 339: 1415-1425.

[3] Benavente O, Eliasziw M, Streifler JY, et al. Prognosis after transient monocular blindness associated with carotid-artery stenosis. N Engl J Med 2001; 345: 1084-1090.

[4] Committee. NS. North American Symptomatic Carotid Endarterectomy Trial. Methods, patient characteristics, and progress. Stroke 1991; 22: 711-720.

[5] Mohr J, Gautier, JC, Pessin, MS. Internal carotid artery disease. In: Barnett HJM, Mohr JP, Stein BM, Yatsu FM, editors. Stroke: pathophysiology, diagnosis, and management. New York: Churchill-Livingstone 1998; 355-400.

[6] Pessin MS, Duncan GW, Mohr JP, et al. Clinical and angiographic features of carotid transient ischemic attacks. N Engl J Med 1977; 296: 358-362.

[7] Inzitari D, Eliasziw M, Sharpe BL, et al. Risk factors and outcome of patients with carotid artery stenosis presenting with lacunar stroke. North American Symptomatic Carotid Endarterectomy Trial Group. Neurology 2000; 54: 660-666.

[8] Mayberg MR, Wilson SE, Yatsu F, et al. Carotid endarterectomy and prevention of cerebral ischemia in symptomatic carotid stenosis. Veterans Affairs Cooperative Studies Program 309 Trialist Group. JAMA 1991; 266: 3289-3294.

[9] Spence JD, Eliasziw M, DiCicco M, et al. Carotid plaque area: a tool for targeting and evaluating vascular preventive therapy. Stroke 2002; 33: 2916-2922.

[10] Adams HP, Jr., Bendixen BH, Kappelle LJ, et al. Classification of subtype of acute ischemic stroke. Definitions for use in a multicenter clinical trial. TOAST. Trial of Org 10172 in Acute Stroke Treatment. Stroke 1993; 24: 35-41.

[11] Chen ZM, Sandercock P, Pan HC, et al. Indications for early aspirin use in acute ischemic stroke: A combined analysis of 40000 randomized patients from the chinese acute stroke trial and the international stroke trial. On behalf of the CAST and IST collaborative groups. Stroke 2000; 31: 12401249.

[12] Grau AJ, Weimar C, Buggle F, et al. Risk factors, outcome, and treatment in subtypes of ischemic stroke: the German stroke data bank. Stroke 2001; 32: 2559-2566. 
[13] Muir KW, Weir CJ, Murray GD, et al. Comparison of neurological scales and scoring systems for acute stroke prognosis. Stroke 1996; 27: 1817-1820.

[14] Yip HK, Liou CW, Chang HW, et al. Link between platelet activity and outcomes after an ischemic stroke. Cerebrovasc Dis 2005; 20: 120-128.

[15] Yip HK, Chang LT, Chang WN, et al. Level and value of circulating endothelial progenitor cells in patients after acute ischemic stroke. Stroke 2008; 39: 69-74.

[16] Yip HK, Tsai TH, Lin HS, et al. Effect of erythropoietin on level of circulating endothelial progenitor cells and outcome in patients after acute ischemic stroke. Crit Care 2011; 15: R40.

[17] Chen YL, Tsai TH, Wallace CG, et al. Intra-carotid arterial administration of autologous peripheral blood-derived endothelial progenitor cells improves acute ischemic stroke neurological outcomes in rats. Int J Cardiol 2015; 201: 668-683.

[18] Sung PH, Lin HS, Lin WC, et al. Intra-carotid arterial transfusion of autologous circulatory derived CD34+ cells for old ischemic stroke patients - a phase I clinical trial to evaluate safety and tolerability. Am J Transl Res 2018; 10: 2975-2989.

[19] Chen $\mathrm{KH}$, Chen $\mathrm{CH}$, Wallace $\mathrm{CG}$, et al. Intravenous administration of xenogenic adipose-derived mesenchymal stem cells (ADMSC) and ADMSC-derived exosomes markedly reduced brain infarct volume and preserved neurological function in rat after acute ischemic stroke. Oncotarget 2016; 7: 74537-74556.

[20] Wagner DC, Riegelsberger UM, Michalk S, et al. Cleaved caspase-3 expression after experimental stroke exhibits different phenotypes and is predominantly non-apoptotic. Brain Res 2011; 1381: 237-242.

[21] Boltze J, Schmidt UR, Reich DM, et al. Determination of the therapeutic time window for human umbilical cord blood mononuclear cell transplantation following experimental stroke in rats. Cell Transplant 2012; 21: 1199-1211.

[22] Grajek S, Popiel M, Gil L, et al. Influence of bone marrow stem cells on left ventricle perfusion and ejection fraction in patients with acute myocardial infarction of anterior wall: randomized clinical trial: Impact of bone marrow stem cell intracoronary infusion on improvement of microcirculation. Eur Heart $\mathrm{J}$ 2010; 31: 691-702.

[23] Traverse $\mathrm{JH}$, Henry TD and Moye LA. Is the measurement of left ventricular ejection fraction the proper end point for cell therapy trials? An analysis of the effect of bone marrow mononuclear stem cell administration on left ventricular ejection fraction after ST-segment elevation myocardial infarction when evaluated by cardiac magnetic resonance imaging. Am Heart J 2011; 162: 671-677.

[24] Leu S, Sun CK, Sheu JJ, et al. Autologous bone marrow cell implantation attenuates left ventricular remodeling and improves heart function in porcine myocardial infarction: an 
echocardiographic, six-month angiographic, and molecular-cellular study. Int J Cardiol 2011; 150: 156168.

[25] Sun CK, Chang LT, Sheu JJ, et al. Bone marrow-derived mononuclear cell therapy alleviates left ventricular remodeling and improves heart function in rat-dilated cardiomyopathy. Crit Care Med 2009; 37: 1197-1205.

[26] Yip HK, Chang LT, Wu CJ, et al. Autologous bone marrow-derived mononuclear cell therapy prevents the damage of viable myocardium and improves rat heart function following acute anterior myocardial infarction. Circ J 2008; 72: 1336-1345.

[27] Yang CC, Chen $\mathrm{YT}$, Chen $\mathrm{CH}$, et al. The therapeutic impact of entresto on protecting against cardiorenal syndrome-associated renal damage in rats on high protein diet. Biomed Pharmacother 2019; 116: 108954.

[28] Yip HK, Chen KH, Dubey NK, et al. Cerebro- and renoprotective activities through platelet-derived biomaterials against cerebrorenal syndrome in rat model. Biomaterials 2019; 214: 119227.

[29] Weise G, Lorenz M, Posel C, et al. Transplantation of cryopreserved human umbilical cord blood mononuclear cells does not induce sustained recovery after experimental stroke in spontaneously hypertensive rats. J Cereb Blood Flow Metab 2014; 34: e1-9.

[30] Wagner DC, Bojko M, Peters M, et al. Impact of age on the efficacy of bone marrow mononuclear cell transplantation in experimental stroke. Exp Transl Stroke Med 2012; 4: 17.

[31] Cui LL, Golubczyk D, Tolppanen AM, et al. Cell therapy for ischemic stroke: Are differences in preclinical and clinical study design responsible for the translational loss of efficacy? Ann Neurol 2019; 86: 5-16.

[32] Balkaya MG, Trueman RC, Boltze J, et al. Behavioral outcome measures to improve experimental stroke research. Behav Brain Res 2018; 352: 161-171.

[33] Cui LL, Kerkela E, Bakreen A, et al. The cerebral embolism evoked by intra-arterial delivery of allogeneic bone marrow mesenchymal stem cells in rats is related to cell dose and infusion velocity. Stem Cell Res Ther 2015; 6: 11.

\section{Supplementary Figure Information}

\section{Supplementary Figure 1. Illustrating the flow cytometric analysis for identification of the EPC surface markers (i.e., endothelial lineage) after 21-day cell culture}
A) Illustrating the flow cytometric analysis for identification of EPC surface markers. B) Illustrating the morphologic feature (400x) of EPCs after 21-day cell culture, i.e., cobblestone-like morphology typical for endothelial cells. C) Expressed the data as mean \pm SD $(n=4)$. 
Supplementary Figure 2. Schematic flow chart showing the time points of preparation EPCs and strategic management for the IS animals among the seven groups

$\mathrm{EPC}=$ endothelial progenitor cell; $\mathrm{SC}=$ sham-operated control

\section{Figures}

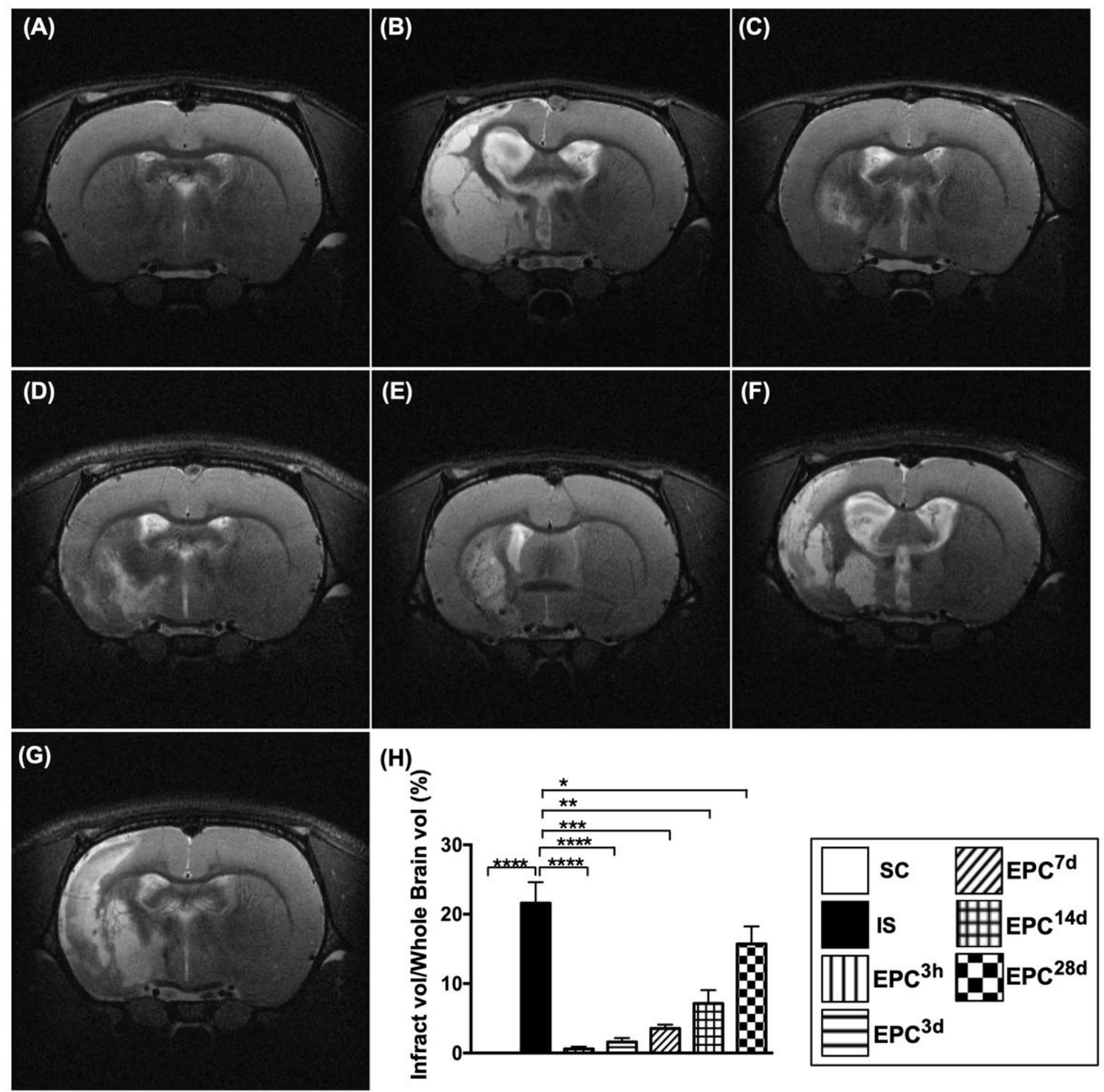

Figure 1 
The brain infarct volume (BIV) at day 60 after acute IS A to G) Illustrating the brain magnetic resonance imaging (MRI) study for identification of brain infarct zone (whitish color)/BIV. H) Analytical result of BIV

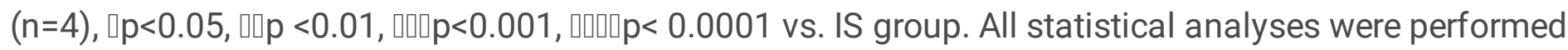
by one-way ANOVA, followed by Bonferroni multiple comparison post hoc test ( $\mathrm{n}=4$ for each group). $\mathrm{Vol}=$ volume. LCCA = left common carotid artery. SC = sham-operated control; IS = ischemic stroke (IS); EPC3h $=$ EPC administration from LCCA at 3h after acute IS; EPC3d $=$ EPC administration from LCCA at day 3 after acute IS; EPC7d = EPC administration from LCCA at day 7 after acute IS; EPC14d = EPC administration from LCCA at day 14 after acute IS; EPC28d = EPC administration from LCCA at day 28 after acute IS.

(A)

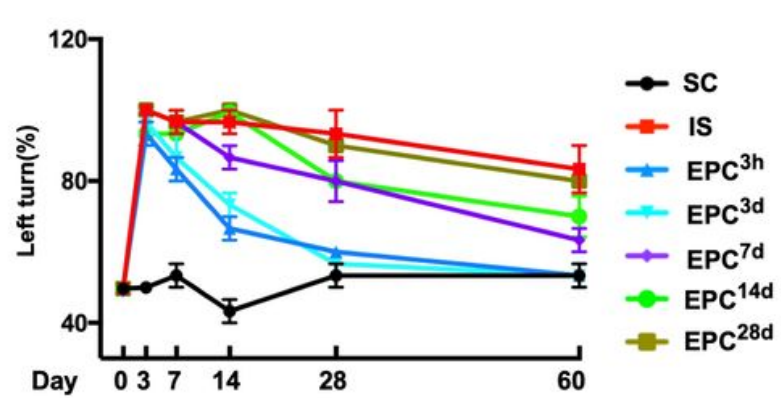

(D)

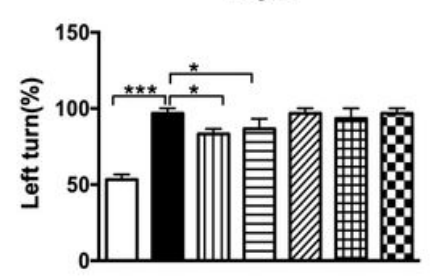

Day 14

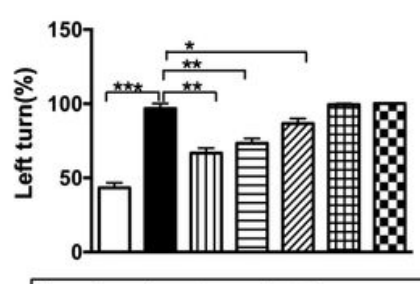

(B)

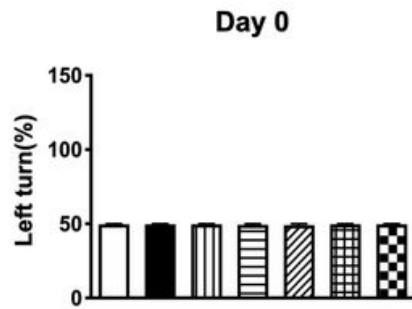

(F)

(E)

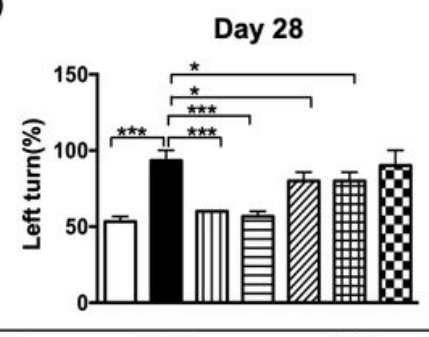

(C)

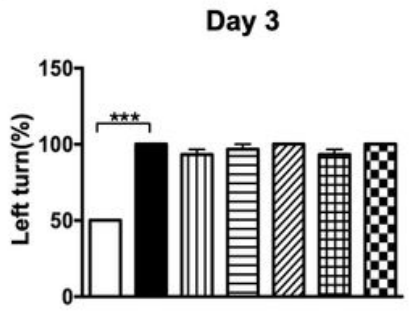

(G)

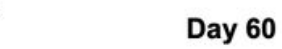

$\square$ sc $\square$ is $\|_{\text {EPc }}^{3 h}$

Figure 2

Time courses of corner test for assessment of neurological function at different time points after acute IS A) Graphical illustration of time points of corner test for determining the neurological function prior to and after acute IS among the seven groups. B) At day $0, p>0.5$. C) At day $3,000<0.001$ vs. SC. D) At day

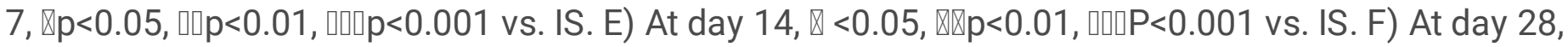

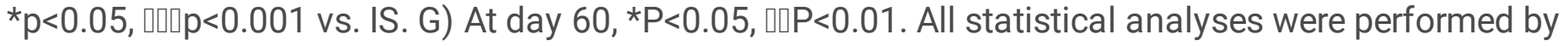
one-way ANOVA, followed by Bonferroni multiple comparison post hoc test ( $\mathrm{n}=10$ for each group). $\mathrm{SC}=$ sham-operated control; IS = ischemic stroke (IS); EPC3h = EPC administration from LCCA at 3h after acute IS; EPC3d = EPC administration from LCCA at day 3 after acute IS; EPC7d = EPC administration from LCCA at day 7 after acute IS; EPC14d = EPC administration from LCCA at day 14 after acute IS; EPC28d $=$ EPC administration from LCCA at day 28 after acute IS. 
(A)

CD31

(83 kDa)

$\beta$-Actin

(43 kDa)

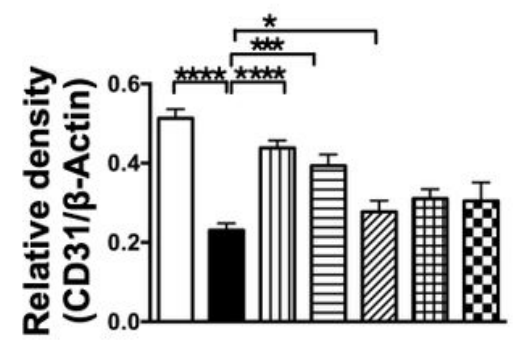

(D)
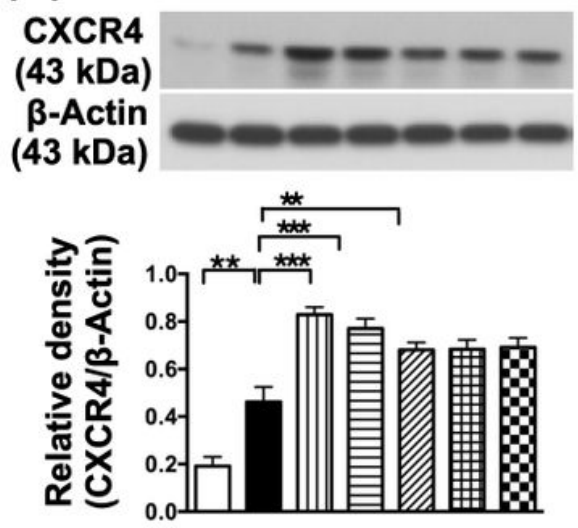

(B)

vWF
(309 kDa)

B-Actin

(43 kDa)
(C)
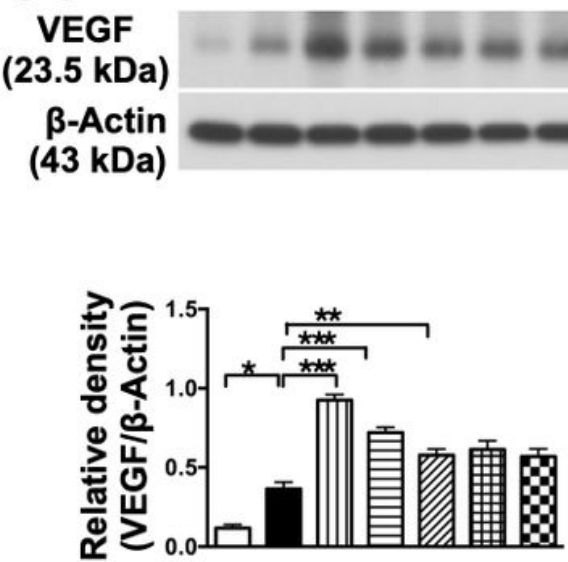

(E)
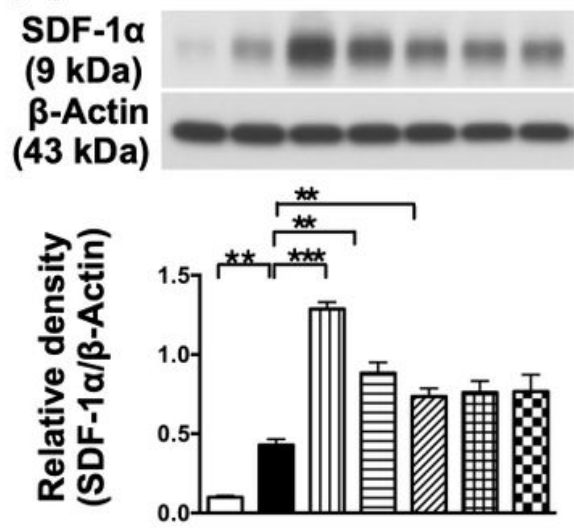

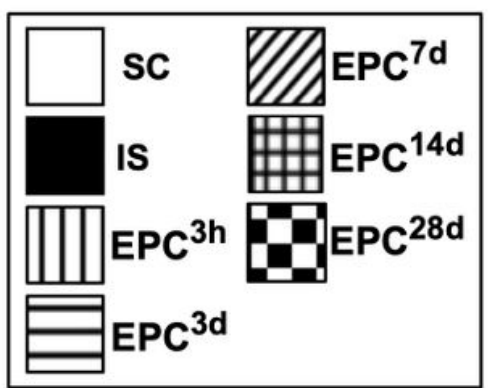

Figure 3

Protein expressions of angiogenesis biomarkers in brain infarct zone by day 60 after acute IS A) Protein

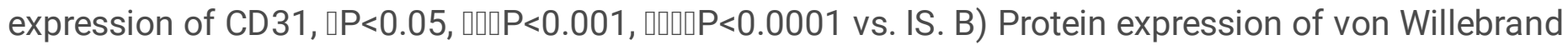

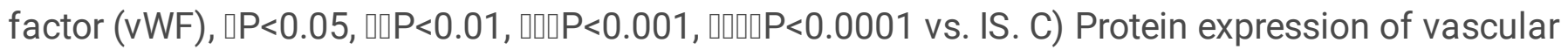

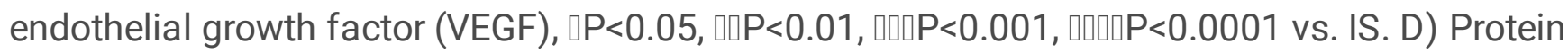

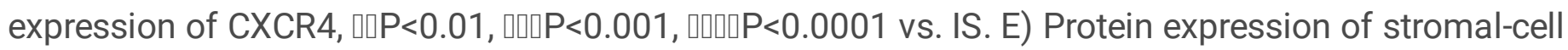

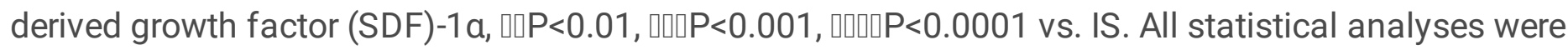
performed by one-way ANOVA, followed by Bonferroni multiple comparison post hoc test $(n=6$ for each group). LCCA = left common carotid artery. SC = sham-operated control; IS = ischemic stroke (IS); EPC3h $=$ EPC administration from LCCA at $3 \mathrm{~h}$ after acute IS; EPC3d = EPC administration from LCCA at day 3 after acute IS; EPC7d = EPC administration from LCCA at day 7 after acute IS; EPC14d = EPC administration from LCCA at day 14 after acute IS; EPC28d = EPC administration from LCCA at day 28 after acute IS. 

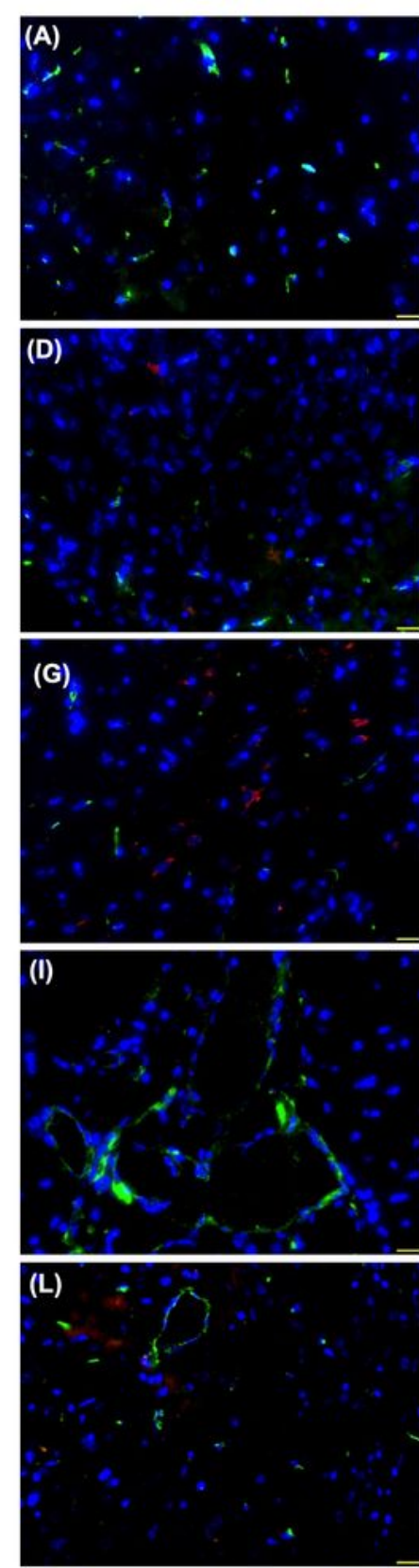

(0)

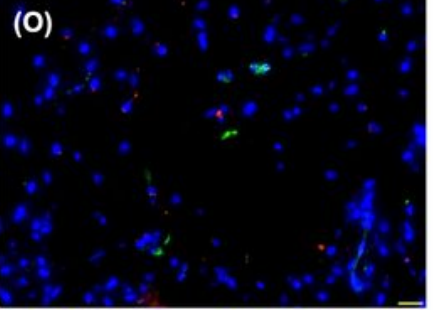

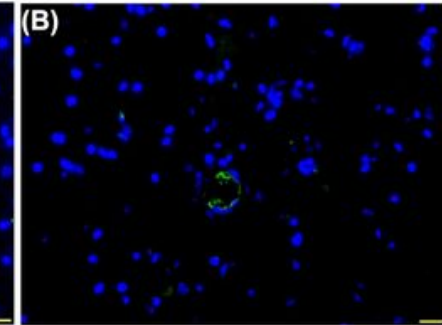

(E)

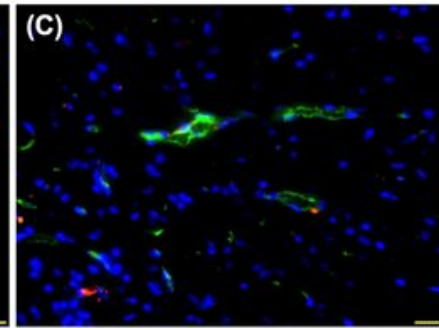

(F)
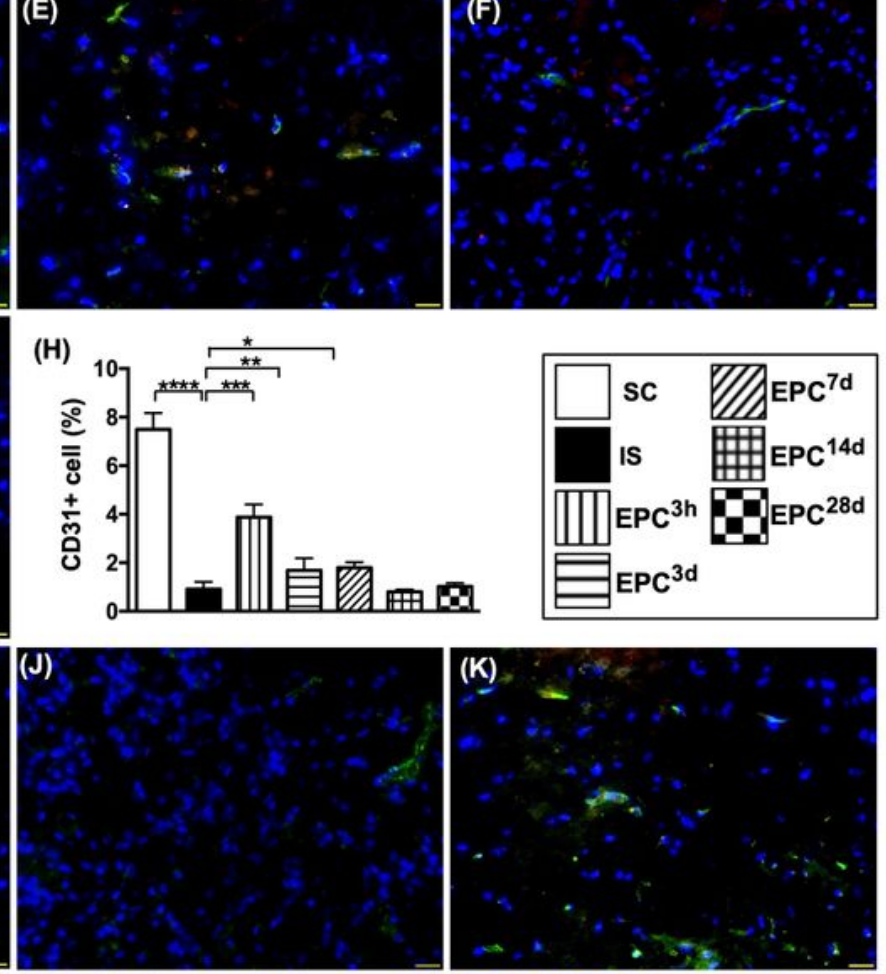

(N)

(M)
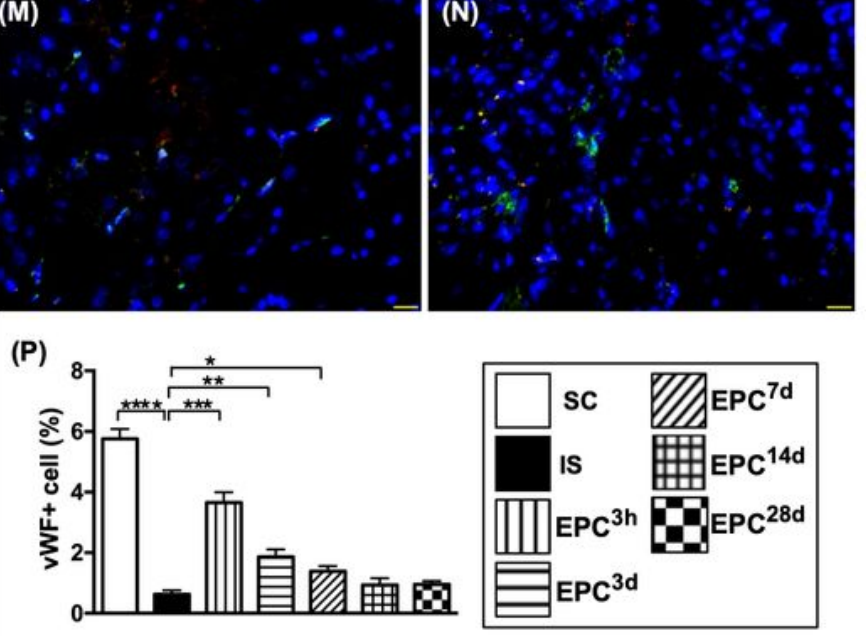

\section{Figure 4}

Expressions of endothelial cell biomarkers in brain infarct zone at day 60 after acute IS A to G) Illustrating immunofluorescent (IF) microscopic finding (400x) for identification of the expression of CD31+ cells (green color). Red color in (C) to (G) indicated the dye-stained implanted ECPs. H) Analytical result of

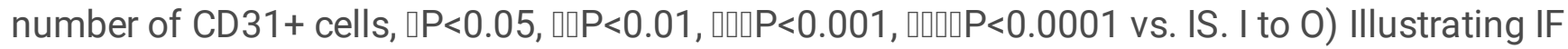
microscopic finding (400x) for identification of the expression of von Willebrand factor (vWF)+ cells 
(green color). Red color in (K) to (O) indicated the dye-stained implanted ECPs. P) Analytical result of

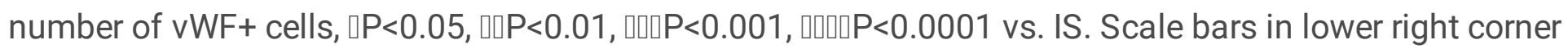
represent $20 \mu \mathrm{m}$. All statistical analyses were performed by one-way ANOVA, followed by Bonferroni multiple comparison post hoc test ( $\mathrm{n}=6$ for each group). $\mathrm{LCCA}=$ left common carotid artery. $\mathrm{SC}=$ shamoperated control; IS = ischemic stroke (IS); EPC3h = EPC administration from LCCA at $3 \mathrm{~h}$ after acute IS; EPC3d = EPC administration from LCCA at day 3 after acute IS; EPC7d = EPC administration from LCCA at day 7 after acute IS; EPC14d = EPC administration from LCCA at day 14 after acute IS; EPC28d = EPC administration from LCCA at day 28 after acute IS. 

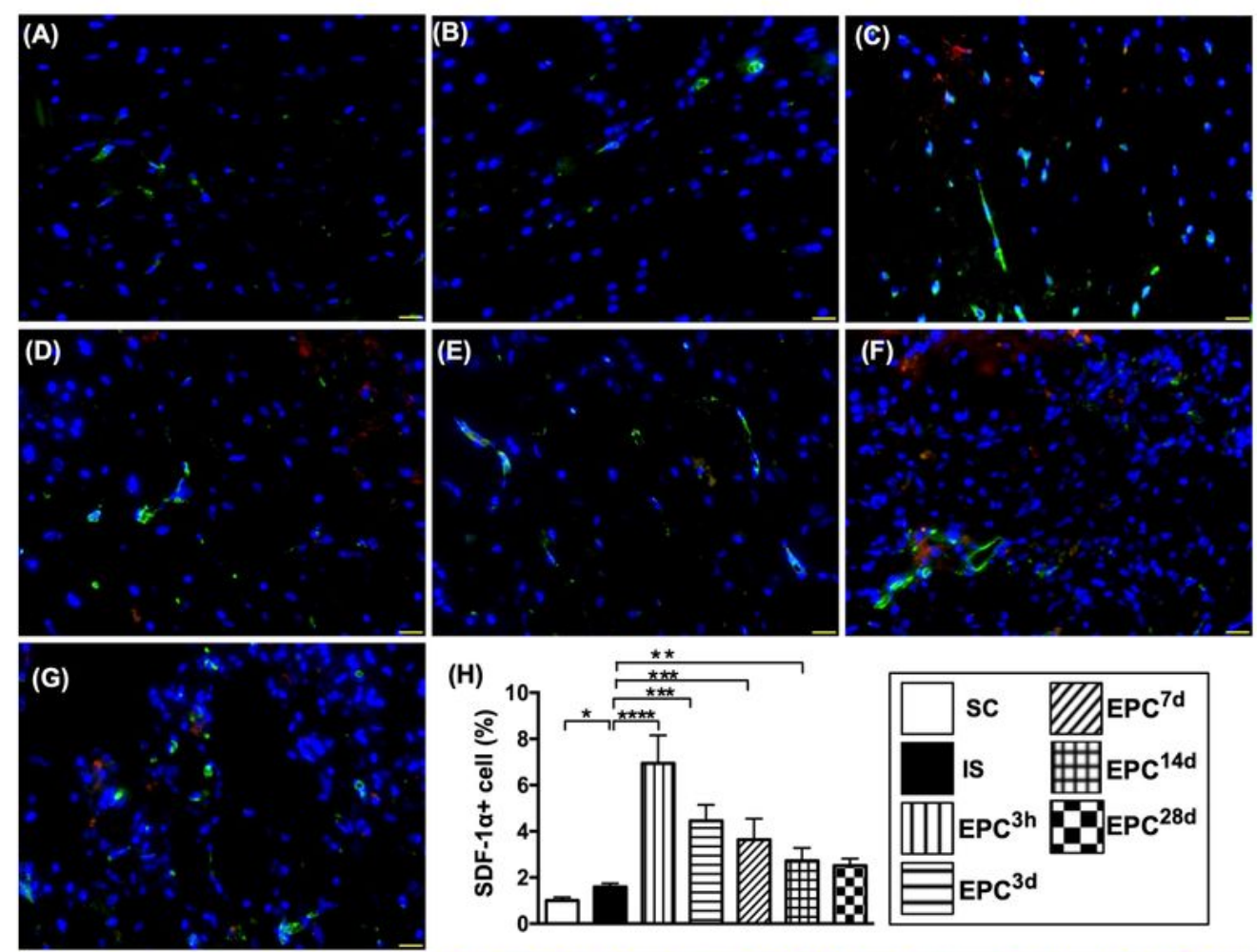

(F)
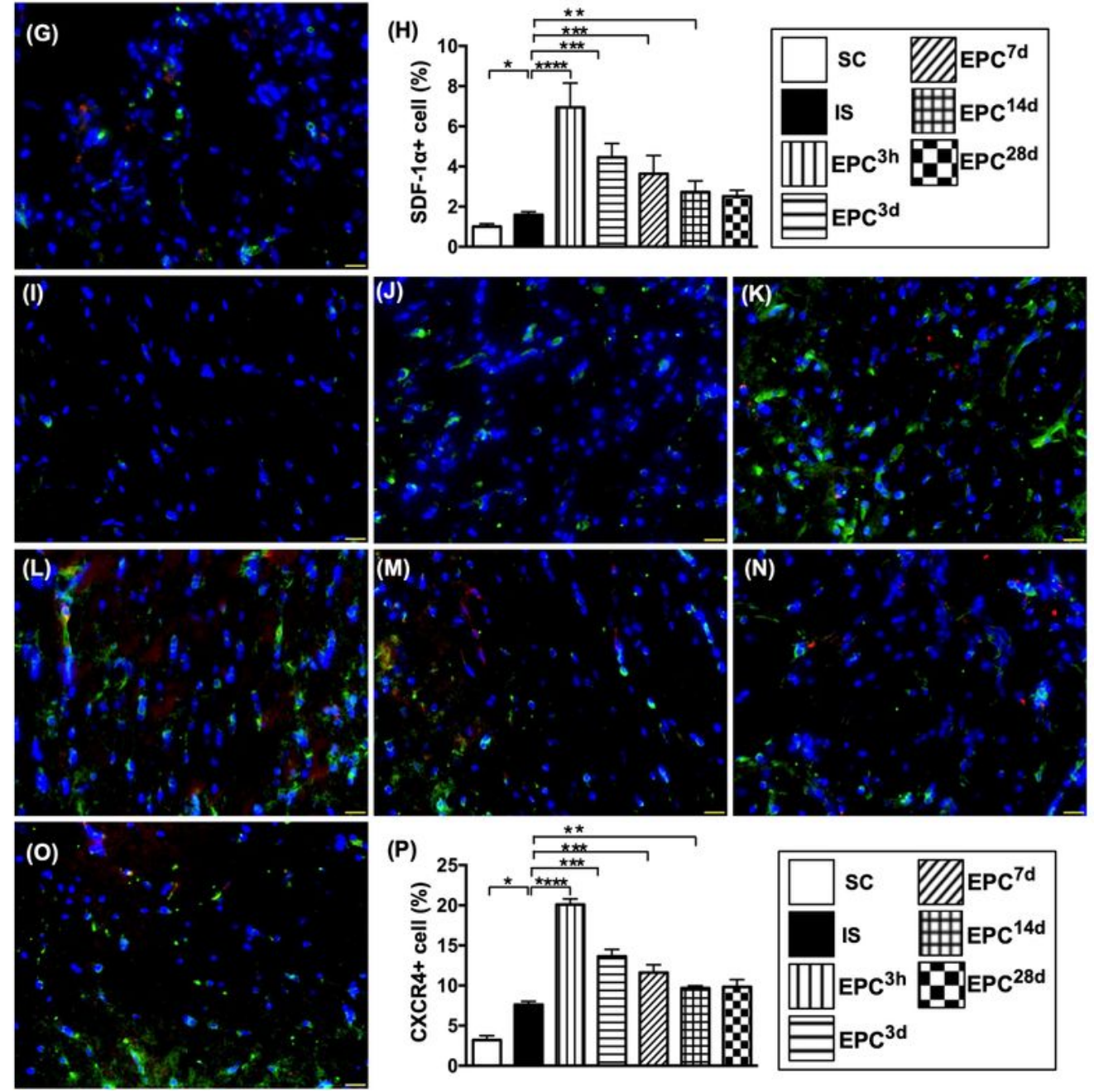

\section{Figure 5}

Cellular expressions of angiogenesis factors in brain infarct zone at day 60 after acute IS A to $\mathrm{G}$ ) Illustrating immunofluorescent (IF) microscopic finding (400x) for identification of the expression of stromal-cell derived growth factor (SDF)-1a+ cells (green color). Red color in (C) to (G) indicated the dyestained implanted ECPs. H) Analytical result of number of SDF-1a+cells, $\square \mathrm{P}<0.05,0 \mathrm{P}<0.01,0 \mathrm{DP}<0.001$,

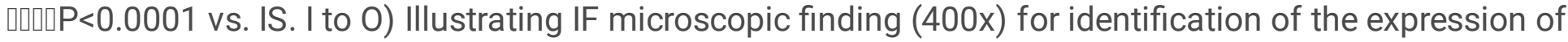


CXCR4+ cells (green color). Red color in (K) to (0) indicated the dye-stained implanted ECPs. P) Analytical

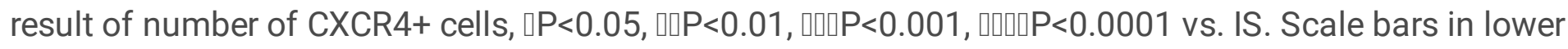
right corner represent $20 \mu \mathrm{m}$. All statistical analyses were performed by one-way ANOVA, followed by Bonferroni multiple comparison post hoc test ( $\mathrm{n}=6$ for each group). LCCA $=$ left common carotid artery. SC = sham-operated control; IS = ischemic stroke (IS); EPC3h = EPC administration from LCCA at $3 \mathrm{~h}$ after acute IS; EPC3d = EPC administration from LCCA at day 3 after acute IS; EPC7d = EPC administration from LCCA at day 7 after acute IS; EPC14d = EPC administration from LCCA at day 14 after acute IS; EPC28d $=$ EPC administration from LCCA at day 28 after acute IS.
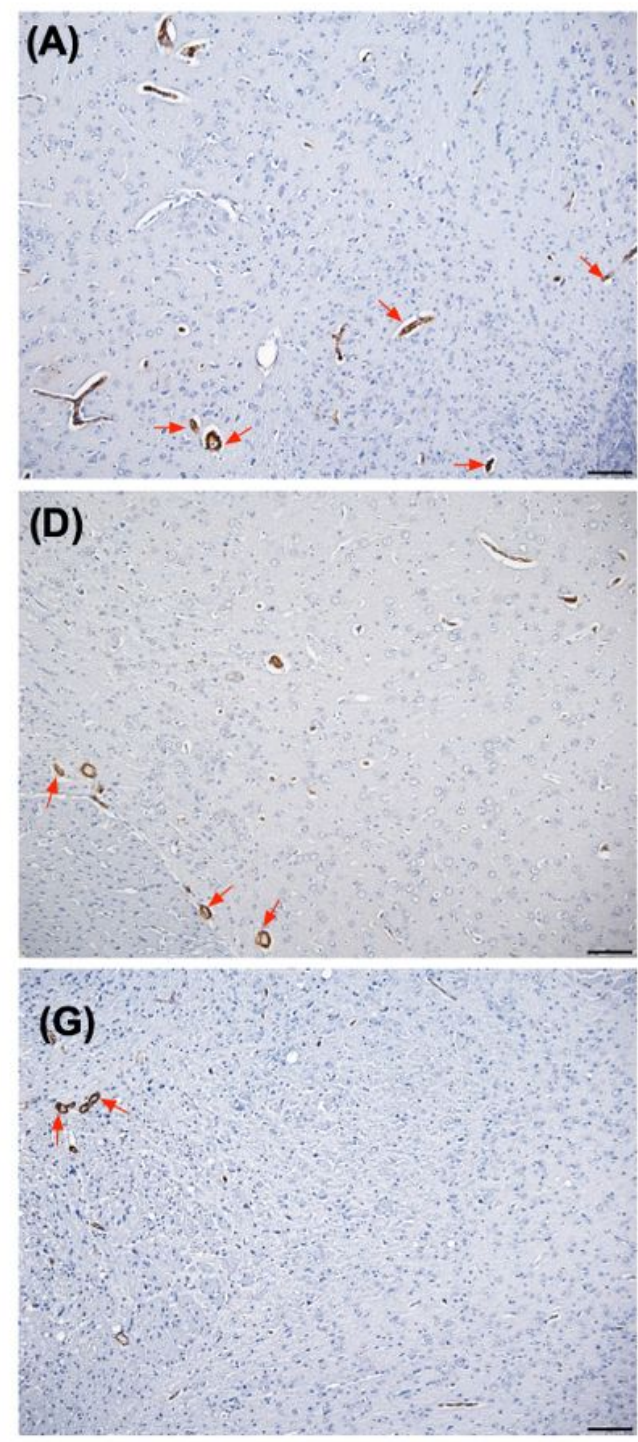

(B)

B)

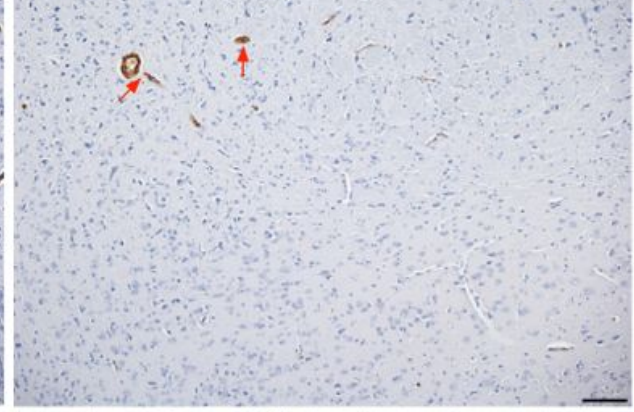

(E)

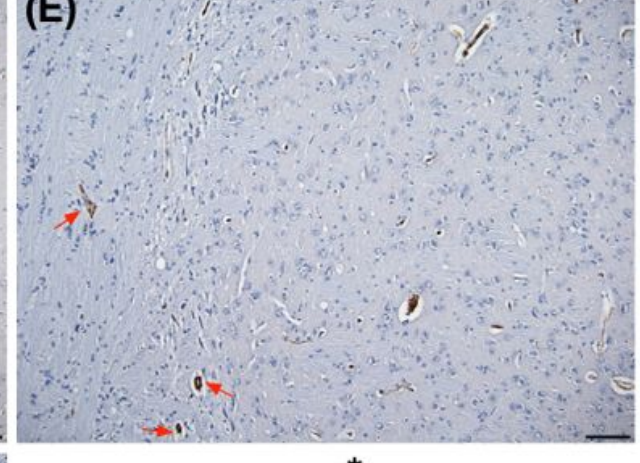

(H)

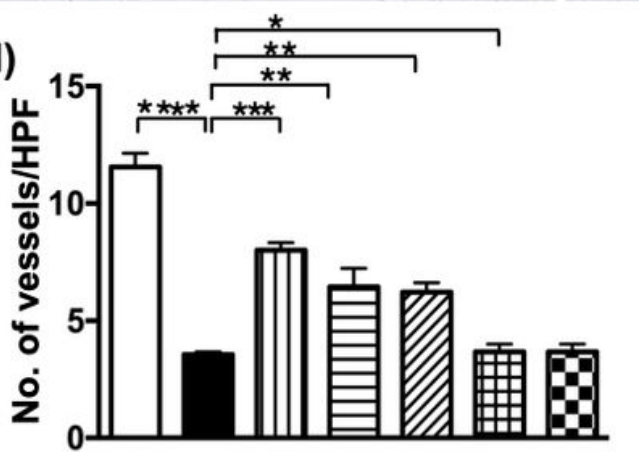

(C)

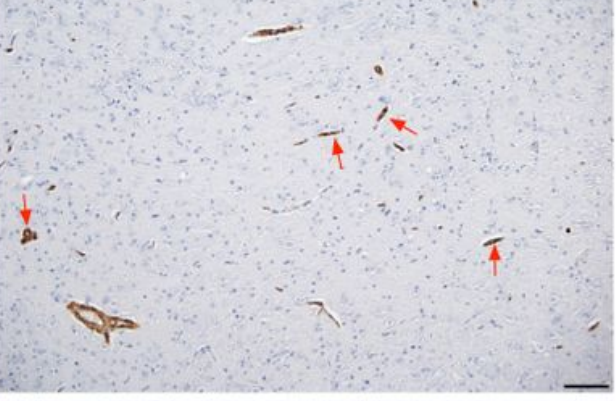

(F)
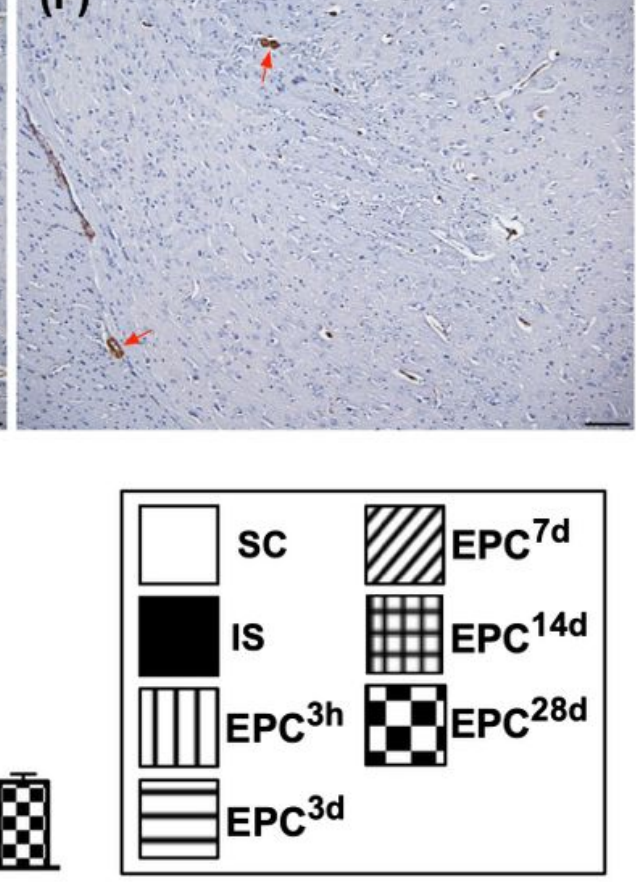

\section{Figure 6}

Expression of small vessel density in brain infarct zone at day 60 after acute IS A to G) Illustrating the microscopic finding (100x) of a-smooth muscle (SMA) staining for identifying the expression of small vessels (i.e., diameter $\leq 25.0 \mu \mathrm{m}$ ) (red arrows). H) Analytical result of number of small vessels, $\square \mathrm{P}<0.05$, $0 \mathrm{P}<0.01,0 . \mathrm{P}<0.001,0.0 \mathrm{P}<0.0001 \mathrm{vs}$. IS. Scale bars in lower right corner represent $100 \mu \mathrm{m}$. All statistical analyses were performed by one-way ANOVA, followed by Bonferroni multiple comparison post hoc test 
( $n=6$ for each group). $\mathrm{LCCA}=$ left common carotid artery. $\mathrm{SC}=$ sham-operated control; IS = ischemic stroke (IS); EPC3h = EPC administration from LCCA at 3h after acute IS; EPC3d = EPC administration from LCCA at day 3 after acute IS; EPC7d = EPC administration from LCCA at day 7 after acute IS; EPC 14d = EPC administration from LCCA at day 14 after acute IS; EPC28d = EPC administration from LCCA at day 28 after acute IS.

(A)

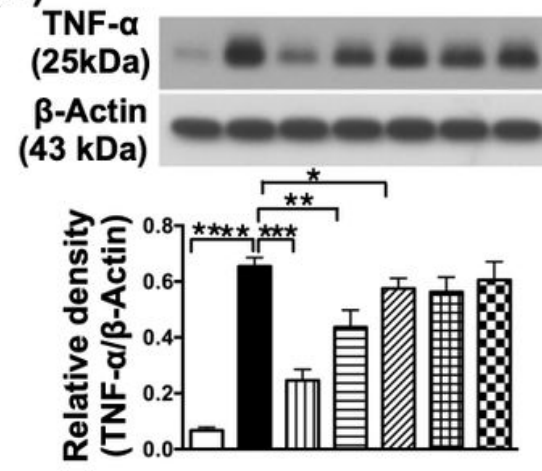

(D)
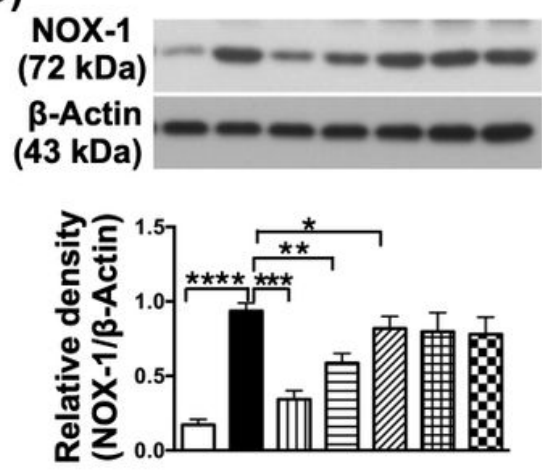

(B)

$$
\begin{gathered}
\text { NF-kB } \\
\text { (65 kDa) } \\
\beta-A c t i n \\
(43 \mathrm{kDa})
\end{gathered}
$$

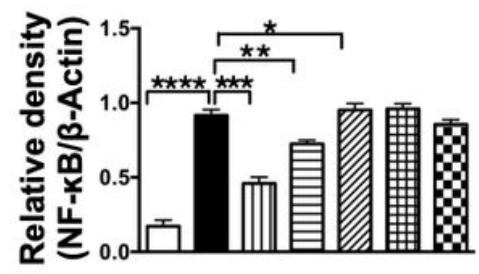

(E)

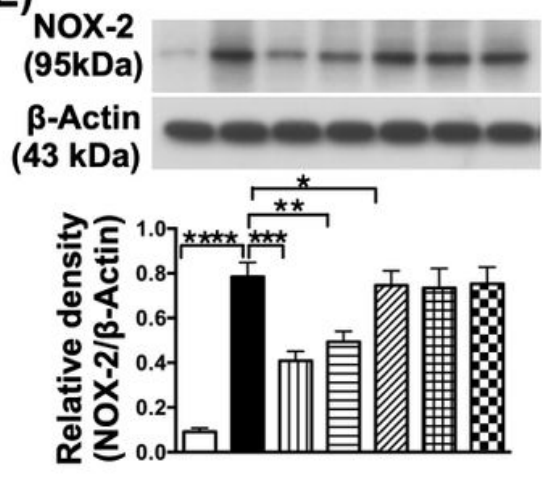

(C)

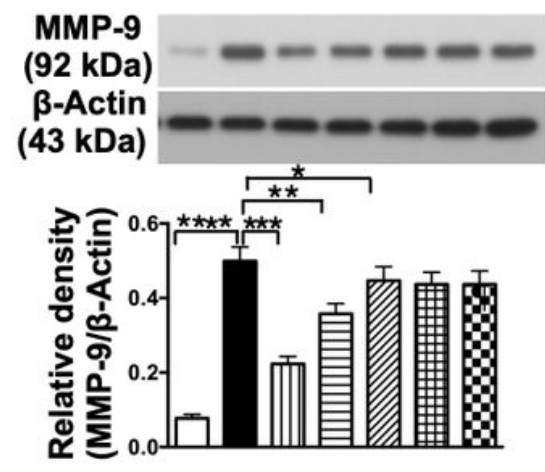

(F)

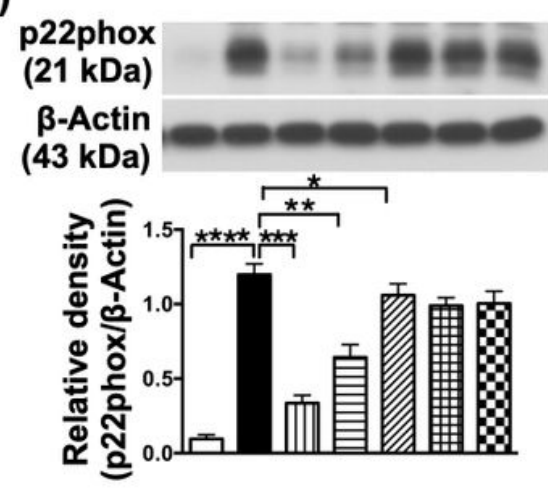

(G)

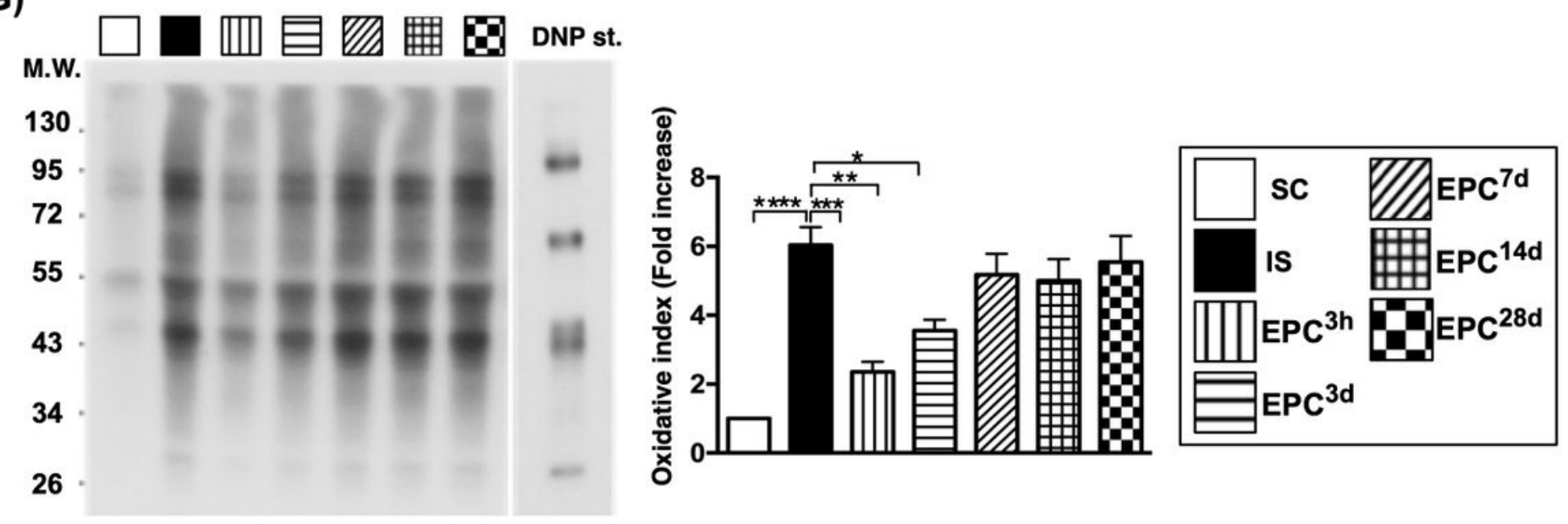

Figure 7

Protein expressions of inflammatory and oxidative stress biomarkers in brain infarct zone at day 60 after acute IS A) Protein expression of tumor necrosis factor (TNF-a), $\mathrm{DP}<0.05, \mathrm{Qu} P<0.01,0 \mathrm{R} P<0.001$,

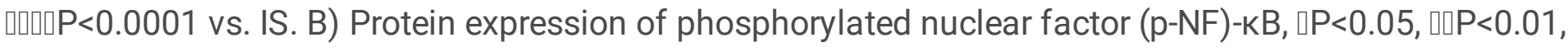




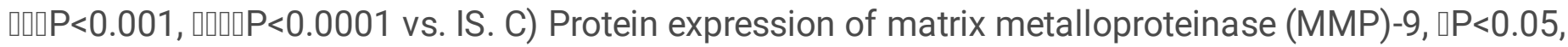

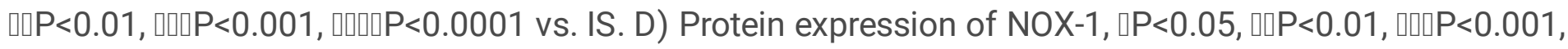

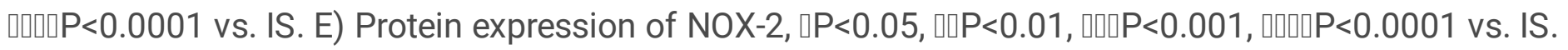

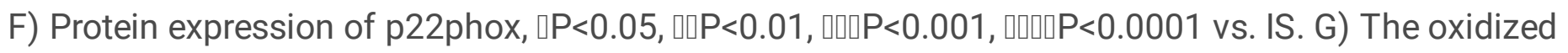

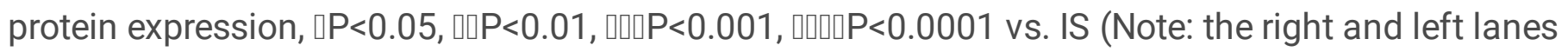
shown on the upper panel represent protein molecular weight marker and control oxidized molecular protein standard, respectively). M.W. = molecular weight; DNP = 1-3 dinitrophenylhydrazone. All statistical analyses were performed by one-way ANOVA, followed by Bonferroni multiple comparison post hoc test ( $n=6$ for each group). LCCA = left common carotid artery. SC = sham-operated control; IS = ischemic stroke (IS); EPC3h = EPC administration from LCCA at 3h after acute IS; EPC3d = EPC administration from LCCA at day 3 after acute IS; EPC7d = EPC administration from LCCA at day 7 after acute IS; EPC14d = EPC administration from LCCA at day 14 after acute IS; EPC28d = EPC administration from LCCA at day 28 after acute IS.

(A)

$$
\begin{aligned}
& \text { Beclin-1 } \\
& (60 \mathrm{kDa})
\end{aligned}
$$

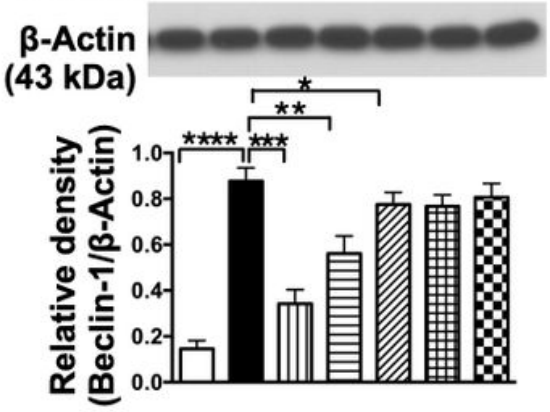

(D)
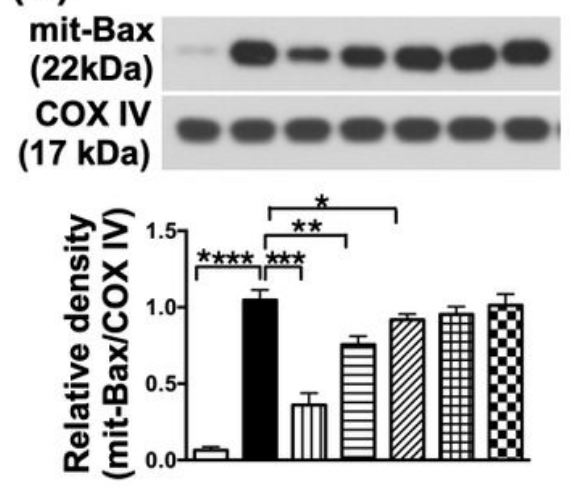

(B)

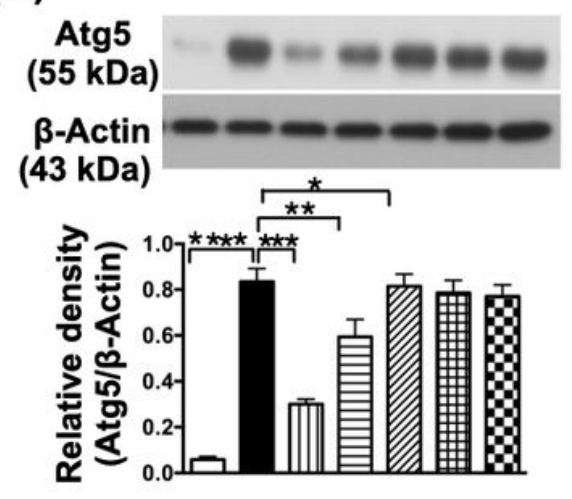

(E)
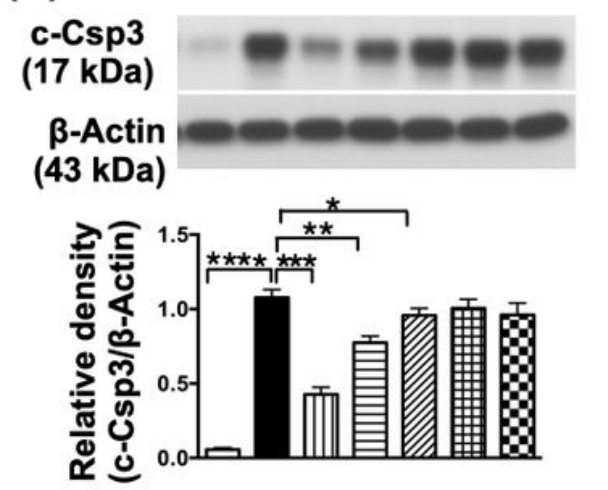

(C)

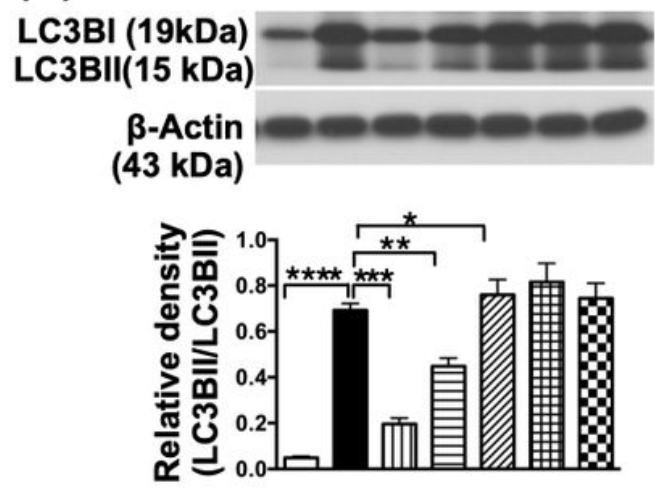

(F)

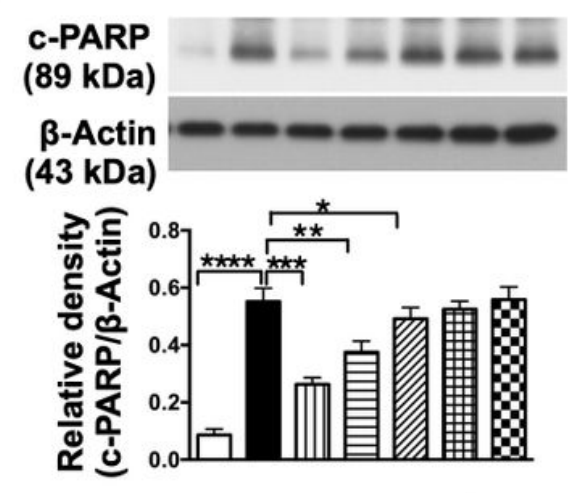

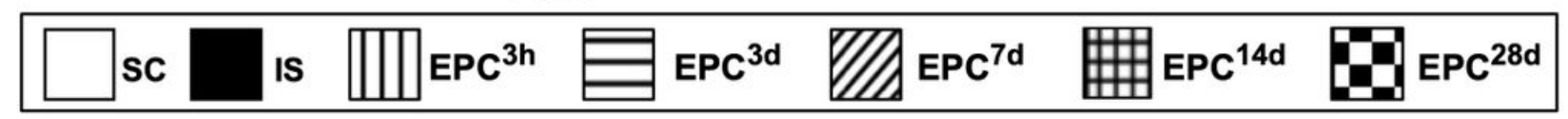

Figure 8

Protein expressions of autophagic and apoptotic biomarkers in brain infarct zone at day 60 after acute IS

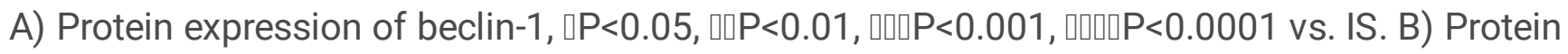

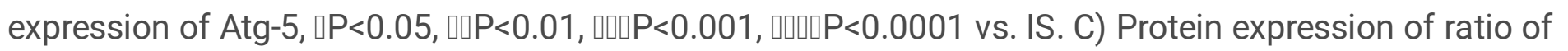

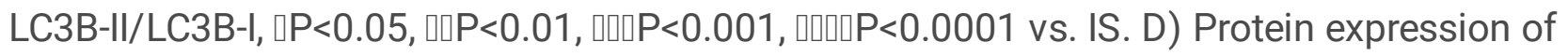




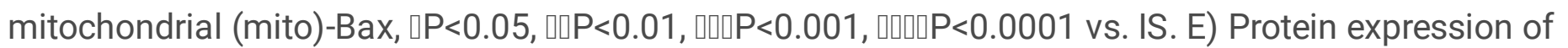

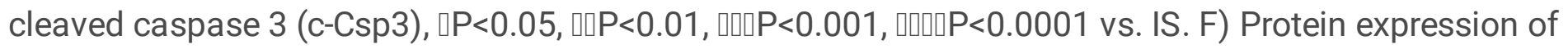

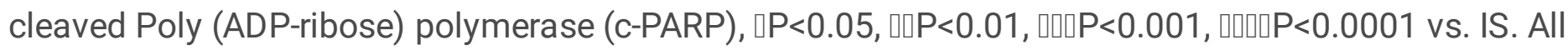
statistical analyses were performed by one-way ANOVA, followed by Bonferroni multiple comparison post hoc test ( $\mathrm{n}=6$ for each group). $\mathrm{LCCA}=$ left common carotid artery. $\mathrm{SC}=$ sham-operated control; IS = ischemic stroke (IS); EPC3h = EPC administration from LCCA at $3 \mathrm{~h}$ after acute IS; EPC3d = EPC administration from LCCA at day 3 after acute IS; EPC7d = EPC administration from LCCA at day 7 after acute IS; EPC14d = EPC administration from LCCA at day 14 after acute IS; EPC28d = EPC administration from LCCA at day 28 after acute IS. 

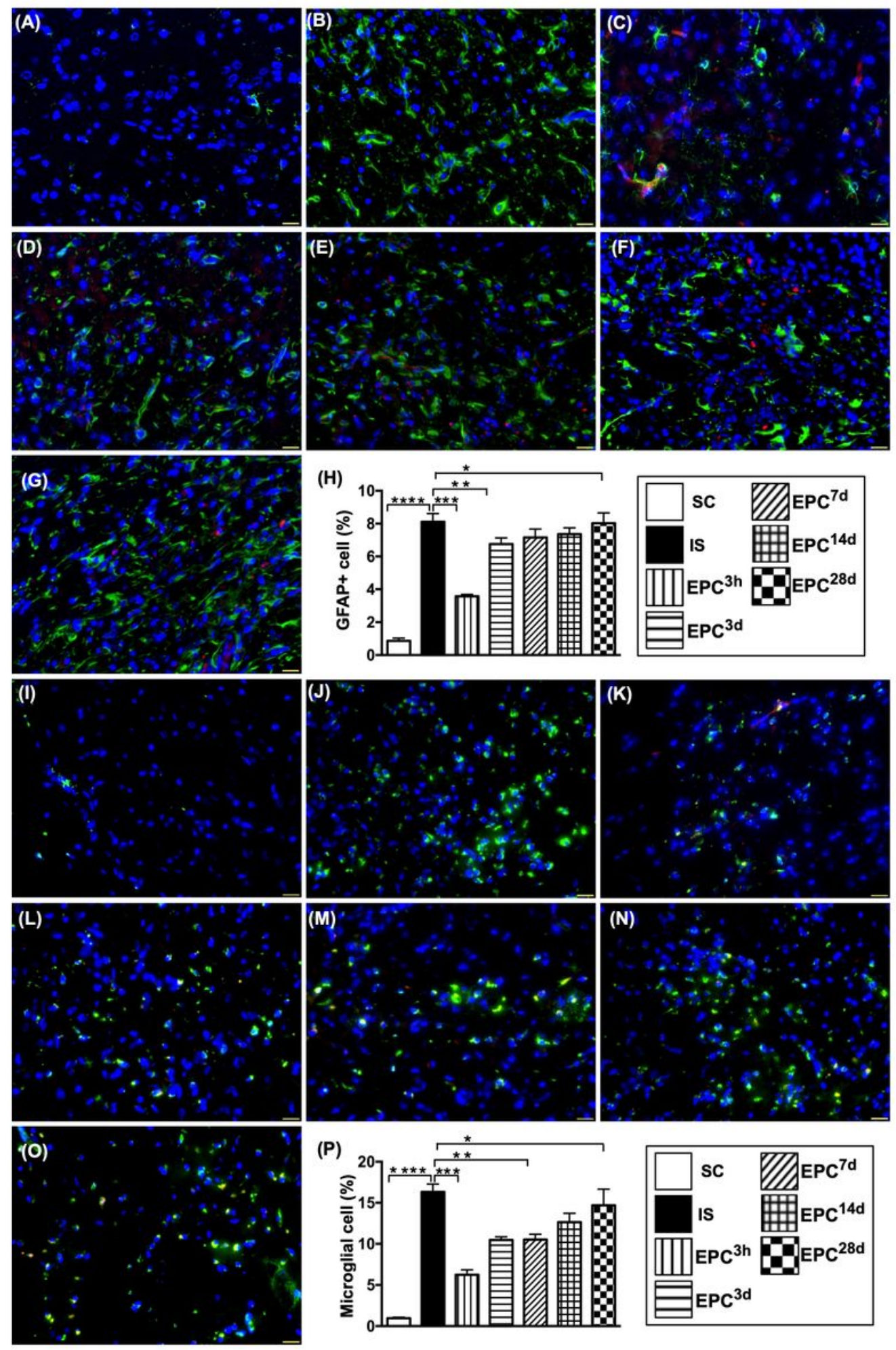

\section{Figure 9}

Inflammatory cell expressions in brain infarct zone at day 60 after acute IS A to G) Illustrating the immunofluorescent (IF) microscopic finding (400x) for identification of positively-stained Glial fibrillary acidic protein (GFAP) cells (green color). Red color in (C) to (G) indicated the dye-stained implanted ECPs.

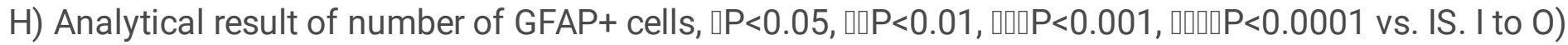
Illustrating the IF microscopic finding (400x) for identification of microglia+ cells (green color). Red color 
in (C) to (G) indicated the dye-stained implanted ECPs. P) Analytical result of number of microglial cells,

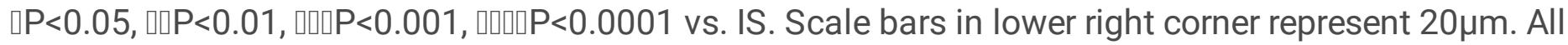
statistical analyses were performed by one-way ANOVA, followed by Bonferroni multiple comparison post hoc test ( $\mathrm{n}=6$ for each group). LCCA = left common carotid artery. $S C=$ sham-operated control; IS = ischemic stroke (IS); EPC3h = EPC administration from LCCA at 3h after acute IS; EPC3d = EPC administration from LCCA at day 3 after acute IS; EPC7d = EPC administration from LCCA at day 7 after acute IS; EPC14d = EPC administration from LCCA at day 14 after acute IS; EPC28d = EPC administration from LCCA at day 28 after acute IS.

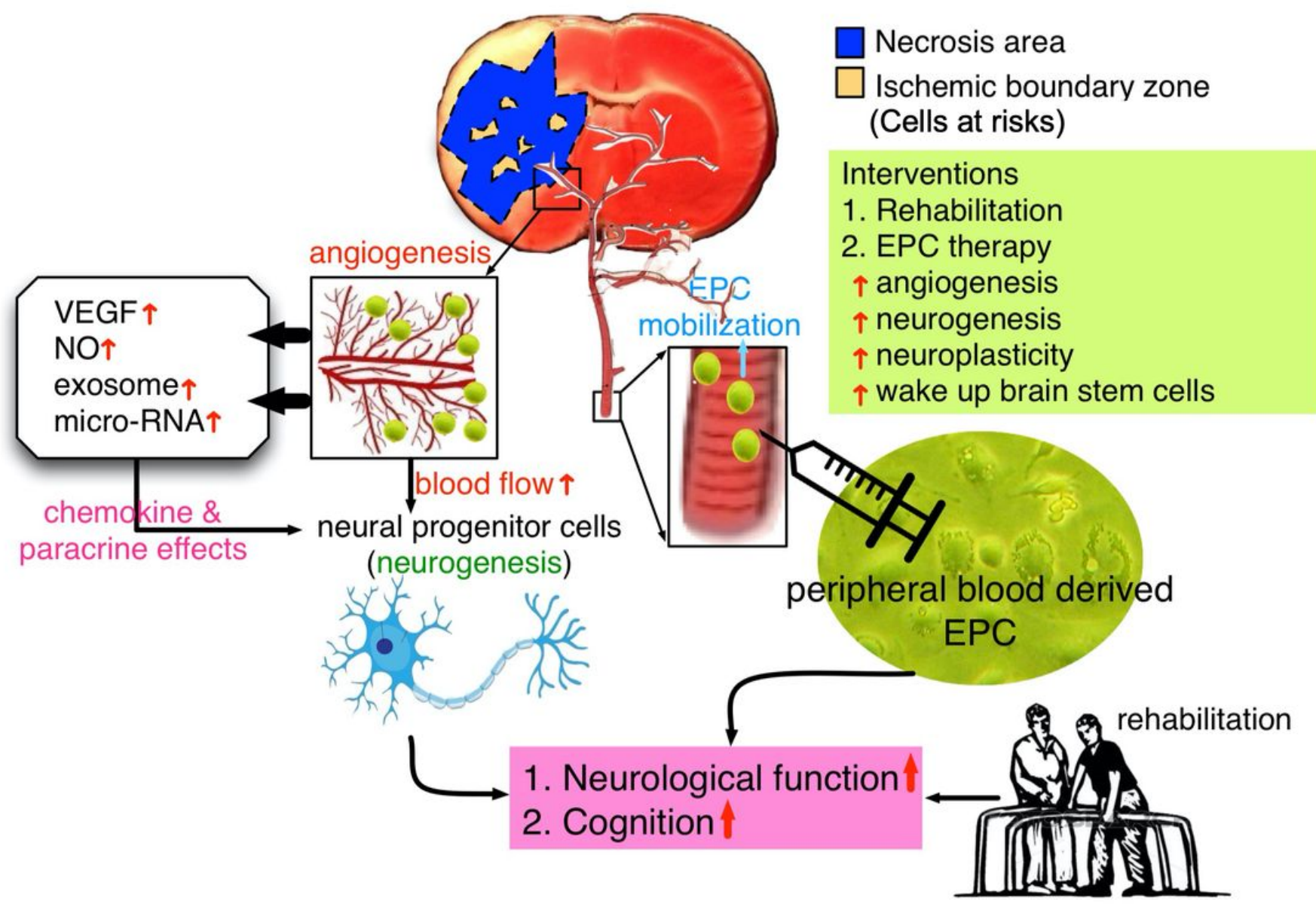

Figure 10

The underlying proposed mechanism of peripheral blood-derived endothelial progenitor cell (EPC) (i.e., CD34+ cells) therapy for improving the outcomes in patients or rodents after acute ischemic stroke VEGF = vascular endothelial growth factors; $\mathrm{NO}=$ nitric oxide.

\section{Supplementary Files}

This is a list of supplementary files associated with this preprint. Click to download. 
- supplementaryFigure1.jpg

- supplementaryFigure2flowchart.jpg 